\title{
Dissipative structures of diffuse molecular gas
}

\section{The translucent environment of a dense core}

\author{
P. Hily-Blant ${ }^{1,2}$ and E. Falgarone ${ }^{1}$ \\ ${ }^{1}$ LRA-LERMA, 24 rue Lhomond, École normale supérieure (Observatoire de Paris), 75231 Paris Cedex 05, France \\ e-mail: hilyblan@iram.fr \\ 2 IRAM, 300 rue de la Piscine, 38406 Saint Martin d'Hères, France
}

Received 22 November 2006 / Accepted 21 February 2007

\begin{abstract}
Aims. This paper belongs to a series of four, dedicated to the analysis of the dynamical, thermal and chemical properties of translucent molecular gas, with the perspective of characterizing the processes driving the dissipation of supersonic turbulence, an anticipated prerequisite of dense core formation.

Methods. We analyze the small scale morphology and velocity structure of the parsec-scale environment of a low mass dense core $\left(1 M_{\odot}\right)$. Our work is based on large maps made with the IRAM-30 m telescope in the two lowest rotational transitions of ${ }^{12} \mathrm{CO}$ and ${ }^{13} \mathrm{CO}$ with high angular $\left(20^{\prime \prime}\right.$ or $0.015 \mathrm{pc}$ at $\left.115 \mathrm{GHz}\right)$ and spectral $\left(0.055 \mathrm{~km} \mathrm{~s}^{-1}\right)$ resolutions. The field is translucent, hence providing strong constraints on the column density and physical conditions in the gas.

Results. More than one third of the field mass $\left(6.5 M_{\odot}\right)$ lies in an elongated tail of dense and cold gas, possibly extending beyond the edge of the map and connected to the core in space and velocity. This core tail is highly turbulent and sub-structured into narrow filaments of aspect ratio up to 20 . These are pure velocity structures with velocity shears in the range $2-10 \mathrm{~km} \mathrm{~s}^{-1} \mathrm{pc}^{-1}$. Another third of the mass, according to the weak extinction of the field, lies in more dilute molecular and atomic gas. Its molecular fraction, largely traced by optically thick ${ }^{12} \mathrm{CO}$ lines, is even more turbulent than the dense core tail. The gas emitting in the broad wings of the ${ }^{12} \mathrm{CO}$ lines is organized into a conspicuous network of narrow criss-crossed filaments, whose pattern at the parsec scale is seen for the first time. The gas there is optically thin in the ${ }^{12} \mathrm{CO}(1-0)$ line $\left(\tau_{12}<0.2\right)$, warmer than $25 \mathrm{~K}$ and more dilute than $1000 \mathrm{~cm}^{-3}$. These optically thin ${ }^{12} \mathrm{CO}$-filaments, though contributing to about $10 \%$ of the mass of the environment, have a CO cooling rate a few times larger than that of the whole field on average. Whether dense or dilute, all the filamentary structures in the field (with transverse sizes $0.015-0.03 \mathrm{pc}$ ), are preferentially oriented along the direction of the magnetic fields, as measured a few parsecs away. Using the Chandrasekhar-Fermi method, we estimate the intensity of the magnetic fields intensity in the dilute molecular gas to be $B_{\text {pos }}=15 \mu \mathrm{G}$. We infer that the turbulent motions in the dilute gas are in the trans-Alfvénic range.

Conclusions. The $1 M_{\odot}$ dense core is surrounded by a translucent and highly turbulent environment whose gas dynamics are not super-Alfvénic. The low mass dense core is not isolated but still connected to a massive reservoir of dense gas. Filaments of optically thin ${ }^{12} \mathrm{CO}$ are found to radiate more efficiently in the $\mathrm{CO}$ lines than the whole field on average. These are the structures that we tentatively identify with the locus of intermittent dissipation of turbulence, and for which there is no observational evidence that they are shocks.
\end{abstract}

Key words. ISM: kinematics and dynamics - ISM: structure - ISM: magnetic fields - line: profiles

\section{Introduction}

Large scale maps of molecular clouds with only moderate star formation provide a comprehensive view of the environment of dense cores before their disruption by young star activity. Whether the tracer is near-IR or visual extinction, molecular lines or dust thermal emission, the coldest and most opaque matter often appears as long filaments in which dense cores are nested (e.g. Abergel et al. 1994; Mizuno et al. 1995; Johnstone et al. 2000; Padoan et al. 2002; Teixeira et al. 2005; Hily-Blant et al. 2005). The open questions raised by star formation therefore now include those of filament formation. Observations of filaments require large maps with a large dynamic to resolve the $0.1 \mathrm{pc}$ typical dense core scale. These observations, now

* Data cubes are only available in electronic form at the CDS anonymous ftp to cdsarc.u-strasbg.fr (130.79.128.5) or via http://cdsweb.u.strasbg.fr/cgi-bin/qcat? J/A+A/469/173 available, in molecular lines, reveal that filaments of cold dense gas are ubiquitous and span a broad range of mass per unit length, from $30-500 M_{\odot} \mathrm{pc}^{-1}$ for massive filaments (Loren 1989a; Onishi et al. 1999; Haikala et al. 2005) down to $0.1 M_{\odot} \mathrm{pc}^{-1}$ (Hily-Blant et al. 2004; Falgarone et al. 1991, 2001) for the most tenuous ones.

Recent observations of the environment of low mass dense cores in ${ }^{12} \mathrm{CO}$ and ${ }^{13} \mathrm{CO}$ rotational transitions, have also brought to light previously hidden velocity structures. The turbulent velocity field of core environments, alike turbulent gas, exhibits more large velocity shears at small scale than anticipated from a Gaussian distribution and the spatial distribution of the nonGaussian occurrences of velocity shears delineates a new kind of small scale structure in these core environments: their locus forms a network of narrow filaments, not coinciding with those of dense gas (Pety \& Falgarone 2003; Hily-Blant 2004; Hily-Blant et al. 2007, hereafter Paper III). It has been shown 
Table 1. Observational characteristics of the field. $\sigma_{X}$ is the average rms, for isotopologue $X$, after regridding on a $10^{\prime \prime}$-grid, with the spectral resolution indicated.

\begin{tabular}{|c|c|c|}
\hline Coordinates..... Gal. & $123.68^{\prime}$ & $24.93^{\prime \prime}$ \\
\hline Coordinates...... Eq. J2000 & $02^{\mathrm{h}} 00^{\mathrm{m}} 06.0^{\mathrm{s}}$ & $87^{\circ} 42^{\prime} 04.1^{\prime \prime}$ \\
\hline Distance......... [pc] & \multicolumn{2}{|c|}{150} \\
\hline Size of the maps.. [pc] & \multicolumn{2}{|c|}{$0.72 \times 0.55$} \\
\hline Number of spectra & \multicolumn{2}{|c|}{7100} \\
\hline Sampling... & \multicolumn{2}{|c|}{10} \\
\hline Spectral resolution $\left[\mathrm{km} \mathrm{s}^{-1}\right]$ & \multicolumn{2}{|c|}{0.055} \\
\hline Frequency ...... [GHz] & 115 & 230 \\
\hline Spatial resolution. ["] & 22 & 11 \\
\hline Linear resolution . [pc] & 0.015 & 0.0075 \\
\hline . $[\mathrm{K}]$ & 0.40 & 0.40 \\
\hline$\sigma_{13} \ldots \ldots \ldots \ldots \ldots[\mathrm{K}]$ & 0.25 & 0.50 \\
\hline
\end{tabular}

by Lis et al. (1996) that they trace extrema of vorticity projected in the plane of the sky. As discussed in Pety \& Falgarone (2003), these velocity structures of large vorticity likely trace either fossil shocks which have generated long-lived vorticity, or genuine coherent $^{1}$ vortices, both manifestations of the intermittence of turbulence dissipation (Pety \& Falgarone 2000). These structures may be the long-searched sites of turbulence dissipation in molecular clouds, as proposed by Joulain et al. (1998).

In an effort to disclose the possible link between the filaments of dense and cold gas and the tenuous velocity filamentary structures, we have extended the ${ }^{12} \mathrm{CO}$ and ${ }^{13} \mathrm{CO}$ maps of a dense core environment to the parsec scale, thus improving the dynamic range compared to that of Falgarone et al. (1998) (hereafter F98) or Pety \& Falgarone (2003). This paper is the second of a series of four, dedicated to the detailed analysis of the parsec scale environment of a low mass dense core, putting into perspective the kinematics, thermal and chemical properties of the filamentary structures disclosed in the field. The peculiar kinematic and chemical signatures of a site of large velocity shear are reported in Falgarone et al. (2006) (hereafter Paper I). High angular observations performed on the same field with the IRAM Plateau de Bure Interferometer are presented in Falgarone et al. (Paper IV, in prep.) and the results of the statistical analysis of the velocity field given in Paper III.

The present paper focuses on the gas properties directly inferred from the CO maps. The observation and data reduction procedures for the large scale mapping are described in Sect. 2. Section 3 is dedicated to the general properties of the spacevelocity structure (maps and line profiles) while Sect. 4 focuses on the size and orientation of the structures identified in the maps. Gas properties are given in Sect. 5. The implications of these results, in terms of gas dynamics and mass distribution of the core environment, are presented in Sect. 6. In Sect. 7, we argue that magnetic fields control the kinematics and morphology of the gas, and discuss the role of turbulence as a heating source for the gas.

\section{Observations and data reduction}

\subsection{Target field}

The target field is located in MCLD 123.5+24.9, a molecular cloud in the Polaris Flare (Heithausen \& Thaddeus 1990, F98). We constrain its distance from the reddening of nearby stars

${ }^{1}$ So-called because their lifetime is significantly longer than their turn-over time. as determined by Hipparcos (Perryman \& ESA 1997). 78 stars closer than $200^{\prime}$ from the dense core were selected from the Hipparcos catalog. The average distance of the reddened stars (weighted by their projected distance to the dense core) is $170 \mathrm{pc}$ which gives an upper limit on the cloud distance. This is consistent with the determination of Zagury et al. (1999), who found 105-125 pc and is also consistent with the upper limit of $240 \mathrm{pc}$ adopted by Heithausen et al. (1993). For consistency with F98 and Heithausen (2002), we adopt $150 \mathrm{pc}$ in the following.

At large scale (Heithausen \& Thaddeus 1990; Grossmann et al. 1990), MCLD 123.5+24.9 is an elongated structure of size $\approx 1.5 \times 0.5 \mathrm{pc}$. It coincides with a maximum of cold dust emission (13 K from Bernard et al. 1999). The extinction, with a spatial resolution of $8^{\prime}$, is $A_{\mathrm{V}} \approx 0.6-0.8 \mathrm{mag}$ (Cambrésy et al. 2001), corresponding to a total hydrogen column density $N_{\mathrm{H}}=1.1-1.5 \times 10^{21} \mathrm{~cm}^{-2}$, given the conversion factor $N_{\mathrm{H}} / A_{\mathrm{V}}=1.87 \times 10^{21} \mathrm{~cm}^{-2} \mathrm{mag}^{-1}$. From the HI column density $N_{\mathrm{HI}}=0.36 \times 10^{21} \mathrm{~cm}^{-2}$ also measured with a $8^{\prime}$ resolution (Heithausen \& Thaddeus 1990), it follows that $N_{\mathrm{H}_{2}}=$ $0.4 \pm 0.1 \times 10^{21} \mathrm{~cm}^{-2}$, so that $N_{\mathrm{H}_{2}} \approx N_{\mathrm{HI}}$, i.e. the fraction of molecular hydrogen is $f=N_{\mathrm{H}_{2}} / N_{\mathrm{H}}=35 \%$ (Heithausen \& Thaddeus 1990).

The region we mapped encompasses a dense core traced by the $\mathrm{C}^{18} \mathrm{O}(1-0)$ line and the $1.2 \mathrm{~mm}$ dust continuum emission as an elongated filament $(0.18 \times 0.03 \mathrm{pc})$ (Gerin et al. 1997; Heithausen 2002). Its properties are comparable to those of dark clouds: the kinetic temperature is low, $6<T_{\text {kin }}<15 \mathrm{~K}$, and the abundances of ${ }^{13} \mathrm{CO}$ and $\mathrm{C}^{18} \mathrm{O}$ have reached their dark clouds values. Further observations, in three $\mathrm{HC}_{3} \mathrm{~N}$ emission lines, by Heithausen (2002) revealed two density maxima located at both ends of the elongated filament. These two entities are dense enough to be gravitationally bound.

\subsection{Observations}

Maps were done with the IRAM-30 m telescope under average summer conditions, between 1999 and 2001. The two lowest rotational transitions of ${ }^{12} \mathrm{CO}$ and ${ }^{13} \mathrm{CO}$ were observed simultaneously. The SSB receiver temperature was $\approx 40-60 \mathrm{~K}$ at $3 \mathrm{~mm}$ and $\approx 120 \mathrm{~K}$ at $1.3 \mathrm{~mm}$. The rejection of the image side band was better than $15 \mathrm{~dB}$ and $10 \mathrm{~dB}$ at $115 \mathrm{GHz}$ and $230 \mathrm{GHz}$ respectively. The corresponding SSB system temperatures were, on average, $\approx 300-350 \mathrm{~K}$ and $\approx 600-750 \mathrm{~K}$. On-the-fly observing mode in total power was used to allow continuous data acquisition as the antenna was moving, with a scanning velocity of $1^{\prime \prime} / \mathrm{s}$. Dumps were done every $4 \mathrm{~s}$ resulting in a spatial sampling of $4^{\prime \prime}$ along the scanning direction. Adjacent rows were spaced by $10^{\prime \prime}$, so the maps are fully-sampled at $3 \mathrm{~mm}\left(22^{\prime \prime} H P B W\right)$ but not at $1.3 \mathrm{~mm}\left(12^{\prime \prime} H P B W\right)$. The maps were decomposed into sub-maps of $4^{\prime} \times 4^{\prime}$. Hot-cold loads calibrations of the gain were done every $15 \mathrm{~min}$. A reference position free of signal (at offsets $2360,-680^{\prime \prime}$ ) was observed before and after each $4^{\prime}$-row during 10 s to subtract the atmospheric contribution. The absolute calibration of the signal is therefore known within 10 and $20 \%$ at low and high frequency, respectively. Each sub-map was scanned in two orthogonal directions $\left(\ell_{\mathrm{II}}\right.$ and $\left.b_{\mathrm{II}}\right)$, and the integration was repeated until we reached a uniform noise over the whole map. The characteristics of the maps are summarized in Table 1. 


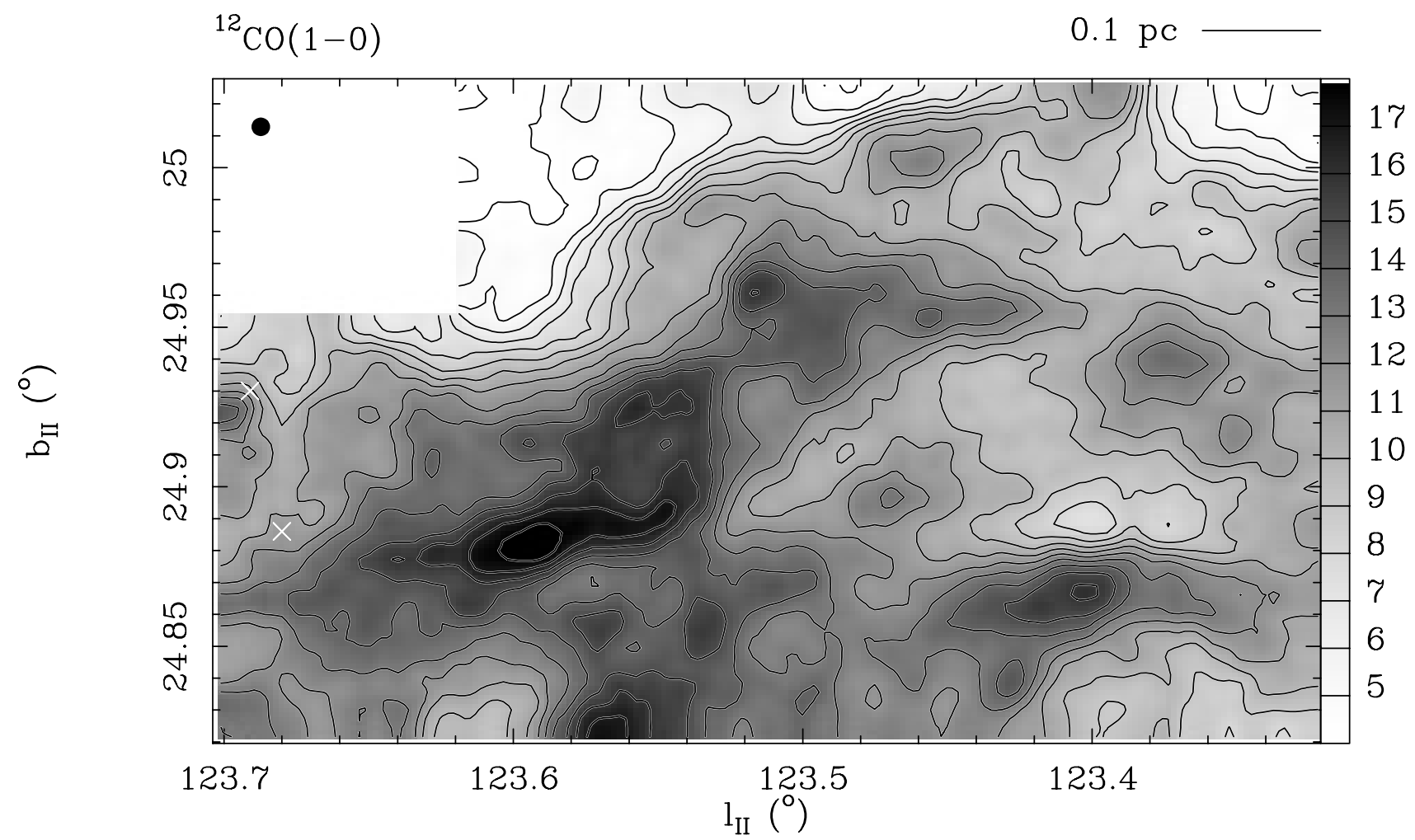

Fig. 1. Integrated intensity $\left(\mathrm{K} \mathrm{km} \mathrm{s}^{-1}, T_{\mathrm{mb}}\right.$ units) maps of the Polaris field, in ${ }^{12} \mathrm{CO}(1-0)$. The $H P B W$ is indicated as a black disk at the upper left corner. The field mapped by Falgarone et al. (1998), which contains the dense core, is located at the East of $\ell_{\mathrm{II}}=123.6^{\circ}$. The white crosses indicate the $\mathrm{HC}_{3} \mathrm{~N}$ peaks (Heithausen 2002). Levels are from 4 to $18 \mathrm{~K} \mathrm{~km} \mathrm{~s}^{-1}$ by step of 1 .

\subsection{Data reduction}

Data were reduced using the GILDAS software ${ }^{2}$. The bandpass of the system was removed, from each spectrum, by subtracting a low-order $(\leq 3)$ polynomial. The spectra were then resampled on a $10^{\prime \prime}$ grid, thus resulting in 7100 spectra data cubes. The $1 \sigma$ noise level, with $0.055 \mathrm{~km} \mathrm{~s}^{-1}$ spectral resolution, is 0.40 and $0.25 \mathrm{~K}$ for the ${ }^{12} \mathrm{CO}$ and ${ }^{13} \mathrm{CO}(1-0)$ lines (see Table 1$)$.

For each tracer, two data cubes corresponding to $\lambda$ and $\beta$ scanning directions are produced, allowing to apply the PLAIT de-stripping algorithm that works in the Fourier domain (Emerson \& Gräve 1988). Stripes, that originate from atmospheric instabilities and instrumental drifts, are unavoidable. If one assumes that stripes present a characteristic length-scale $\delta_{k}$ (where $k$ stands for the scanning direction, either $\lambda$ or $\beta$ ), then the Fourier transform of a map, done in one direction, will present a central band of "corrupted" signal, perpendicular to the direction of the stripes in the direct image, whose characteristic width is $\tilde{\delta_{k}} \sim 1 / \delta_{k}$. Therefore, by merging the weighted Fourier transforms of the two maps done in $\lambda$ and $\beta$, one is able to recover uncorrupted signal, apart from a central region ${ }^{3}$ of size $\approx \tilde{\delta}_{\lambda} \times \tilde{\delta}_{\beta}$. The final data cube is obtained by computing the inverse Fourier transform of the merged Fourier cubes.

When converting the data from $T_{\mathrm{A}}^{*}$ to $T_{\mathrm{mb}}$ we took advantage of large-scale public data (public KOSMA data) to subtract the signal collected in the error-beam of the antenna. We have used the beam characteristics given in Greve et al. (1998). The method is similar to that used by F98 and explained in more

\footnotetext{
${ }^{2}$ URL: http://wWw.iram.fr/IRAMFR/GILDAS/

3 Though the scanning directions need not be orthogonal, this is the optimal choice of pair of directions. In this case, the unrecoverable region is a rectangle in the Fourier plane.
}

details in Bensch et al. (2001). In the following, all main beam temperatures are indeed error-beam-corrected main beam temperatures $^{4}$.

\section{Results}

\subsection{Brightness distribution}

We merged the error-beam-corrected new datasets with those of F98 $\left(\ell_{\mathrm{II}}>123.62^{\circ}\right)$. The cross calibration is very good, as can be seen in the resulting integrated intensity maps of ${ }^{12} \mathrm{CO}$ and ${ }^{13} \mathrm{CO}(1-0)$ shown in Figs. 1 and 2, where both datasets connect smoothly.

Unlike what is often found in ${ }^{12} \mathrm{CO}(1-0)$ emission maps with coarser spatial resolution, a wealth of structures is visible in the integrated intensity map of ${ }^{12} \mathrm{CO}(1-0)$, with a contrast of $\approx 4$ between the brightest and weakest regions. Even higher contrast $(>10)$ is seen in the ${ }^{13} \mathrm{CO}$ integrated map of Fig. 2. The brightest region, to the East of the map is that of the dense core, located between the two $\mathrm{HC}_{3} \mathrm{~N}$ peaks marked with crosses.

At offsets $\delta \ell_{\mathrm{II}}<-500^{\prime \prime}$, the integrated emission of ${ }^{13} \mathrm{CO}(1-0)$ is the strongest where that of ${ }^{12} \mathrm{CO}$ is the weakest (Fig. 3, top panel). This is not due, as might be expected, to self-absorption of the ${ }^{12} \mathrm{CO}$ lines but to a decrease of the ${ }^{12} \mathrm{CO}$ linewidths (see bottom panel). The two most prominent structures in the equivalent width map of Fig. 3 (defined as $\left.\Delta v_{\text {eq }}=\int T_{\text {mb }} \mathrm{d} v / T_{\text {peak }}\right)$ are also clearly visible in the spatial distribution of the ${ }^{12} \mathrm{CO}$ integrated intensity and the variation of $\approx 2$ between the largest and smallest ${ }^{12} \mathrm{CO}$ equivalent width makes the ${ }^{12} \mathrm{CO}$ integrated intensity vary by the same factor because the peak line temperatures are the same. The bright ${ }^{13} \mathrm{CO}$ elongated

\footnotetext{
${ }^{4}$ Data cubes are available in electronic form at the CDS.
} 


\section{$0.1 \mathrm{pc}$}

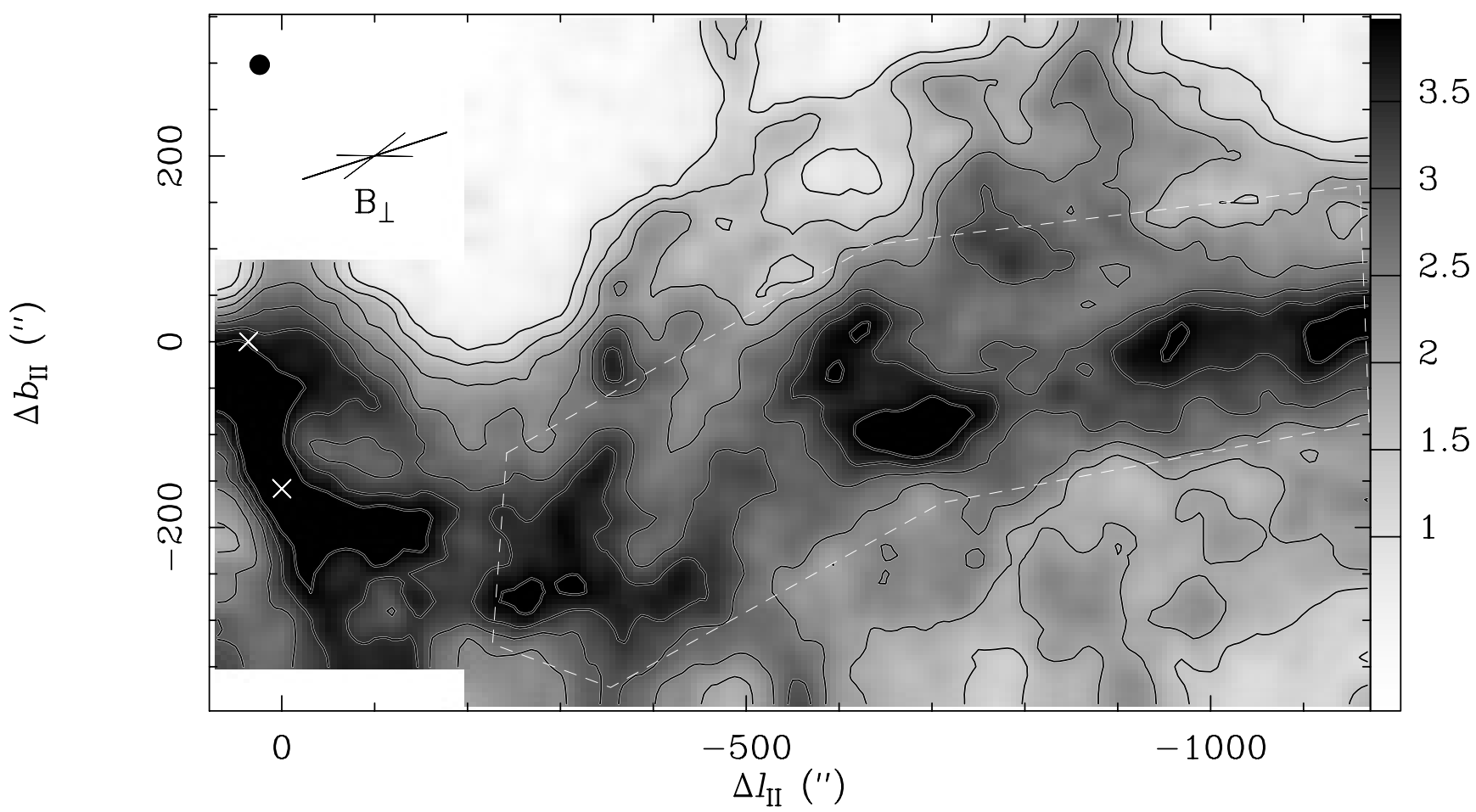

Fig. 2. Same as Fig. 1 for the ${ }^{13} \mathrm{CO}(1-0)$ line. Levels are from 1 to $4 \mathrm{~K} \mathrm{~km} \mathrm{~s}^{-1}$ by step of 0.5 . The orientation of the projection of the magnetic field (measured $4 \mathrm{pc}$ north) is indicated in the upper left corner (thick line) with the $1 \sigma$ uncertainty (thin line) (from Heiles 2000). The dashed white line delineates the region used to derive the gas properties in the main ${ }^{13} \mathrm{CO}$ structure (see text).
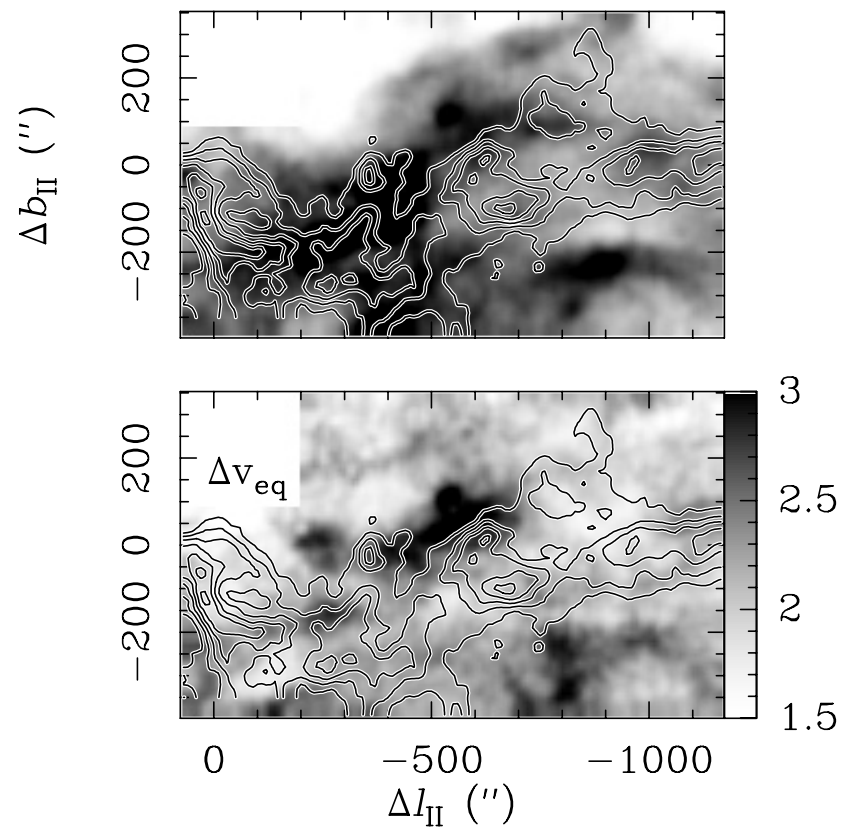

Fig. 3. ${ }^{12} \mathrm{CO}(1-0)$ integrated intensity (top panel, grey scale) and equivalent width $\left(\Delta v_{\mathrm{eq}}\right.$, in $\mathrm{km} \mathrm{s}^{-1}$, bottom panel) with the contours of the ${ }^{13} \mathrm{CO}(1-0)$ integrated intensity overplotted (from 2.5 to $5 \mathrm{~K} \mathrm{~km} \mathrm{~s}^{-1}$, by step of $0.5 \mathrm{~K} \mathrm{~km} \mathrm{~s}^{-1}$ ).

structure therefore coincides, in projection, with a region where the ${ }^{12} \mathrm{CO}$ lines are twice narrower than in the rest of the field. Its half-power diameter, defined as the average value measured along several transverse cuts, is $D \approx 150-200^{\prime \prime}$ or $0.11-0.15 \mathrm{pc}$.
Table 2. Characteristics of the average spectra shown in Fig. 5.

\begin{tabular}{lccc}
\hline \hline Line & $\begin{array}{c}T_{\text {peak }} \\
\mathrm{K}\end{array}$ & $\begin{array}{c}W \\
\mathrm{~K} \mathrm{~km} \mathrm{~s}^{-1}\end{array}$ & $\begin{array}{c}\Delta v_{\text {eq }} \\
\mathrm{km} \mathrm{s}^{-1}\end{array}$ \\
\hline${ }^{12} \mathrm{CO}(1-0)$ & 4.3 & 10.9 & 2.5 \\
${ }^{12} \mathrm{CO}(2-1)$ & 2.7 & 6.5 & 2.5 \\
${ }^{13} \mathrm{CO}(1-0)$ & 1.6 & 2.4 & 1.6 \\
${ }^{13} \mathrm{CO}(2-1)$ & 0.8 & 1.1 & 1.4 \\
\hline
\end{tabular}

The histograms of the ${ }^{12} \mathrm{CO}$ and ${ }^{13} \mathrm{CO}(1-0)$ peak temperature in the field are displayed in Fig. 4. Unlike that of ${ }^{13} \mathrm{CO}$, the ${ }^{12} \mathrm{CO}$ histogram exhibits two maxima at $T_{\text {peak }}=2.5$ and $5 \mathrm{~K}$. The points populating the weak ${ }^{12} \mathrm{CO}$ component (from 1 to $4 \mathrm{~K}$ ), correspond to the regions (in the North-East) or velocity intervals (see next section) devoid of ${ }^{13} \mathrm{CO}$ emission above the noise level.

\subsection{Average profiles}

Figure 5 shows the mean spectra over the whole region, for both isotopologues and both transitions. Their average properties are summarized in Table 2. All averaged spectra are smooth, a characteristic of emission lines in molecular clouds already noted and discussed by Martin et al. (1984) and Tauber et al. (1991). The full velocity extent of the ${ }^{12} \mathrm{CO}$ lines (about $5-6 \mathrm{~km} \mathrm{~s}^{-1}$ ) is significantly larger than that of the ${ }^{13} \mathrm{CO}$ lines $\left(3-4 \mathrm{~km} \mathrm{~s}^{-1}\right)$. The combination of the good signal to noise ratio and the large number of spectra shows that there are velocity intervals in the ${ }^{12} \mathrm{CO}$ lines where ${ }^{13} \mathrm{CO}$ is not detected above the noise level. These intervals will be called the far wings of the ${ }^{12} \mathrm{CO}$ lines, to distinguish them from the core of the line where both tracers 


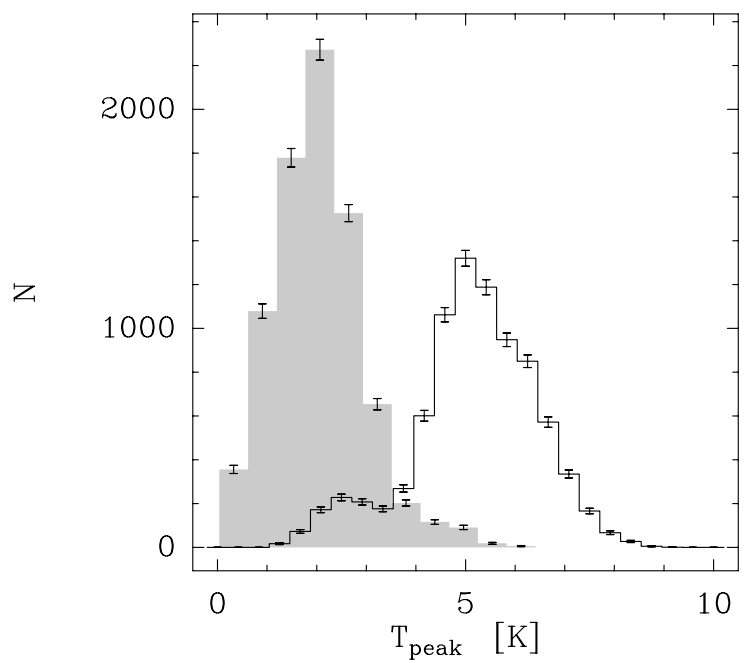

Fig. 4. Histograms of peak temperature. Full line is ${ }^{12} \mathrm{CO}(1-0)$, and light-grey is the ${ }^{13} \mathrm{CO}(1-0)$. Error bars give the statistical uncertainty $(\sqrt{N})$.

are detected. The far wings correspond to velocities in the intervals $[-6.5:-5.5]$ and $[-2:-0.5] \mathrm{km} \mathrm{s}^{-1}$ of the integrated spectra, hereafter referred to as the blue and red wings respectively. The far wing emission that corresponds to ${ }^{12} \mathrm{CO}(1-0)$ line intensities weaker than $\approx 2 \mathrm{~K}$ contributes to the lower peak in the histogram of Fig. 4: it has a distribution in the field distinct from that of the bright lines. The properties of the gas responsible for the far wings, its spatial distribution, density, temperature, kinematics and dynamics is one of the main focus of the present paper.

\subsection{Channel maps}

Maps of the ${ }^{12} \mathrm{CO}$ and ${ }^{13} \mathrm{CO}(1-0)$ emissions integrated over the same velocity intervals of $0.2 \mathrm{~km} \mathrm{~s}^{-1}$ are shown in Fig. 6 . Emission in the far ${ }^{12} \mathrm{CO}$ line wings is seen in the channels where ${ }^{13} \mathrm{CO}$ is not detected at a $3 \sigma$ level $(0.4 \mathrm{~K})$ at $v>-2 \mathrm{~km} \mathrm{~s}^{-1}$ and $v<-5.5 \mathrm{~km} \mathrm{~s}^{-1}$. This emission is emphasized in Fig. 7 which displays the integrated intensity of the red and blue wings separately: it is not uniformly distributed in the map but delineates structures that will be discussed in Sect. 4.

The ${ }^{13} \mathrm{CO}(1-0)$ emission (Fig. 6) is also highly structured in space and velocity, down to the resolution of the observations. It is not straightforward, from these channel maps, to distinguish between pure velocity structure and density structure. For instance, the narrow arc-like emission centered at $v=-4.2 \mathrm{~km} \mathrm{~s}^{-1}$ and running from $\left(-6.7,1.7^{\prime}\right)$ to $\left(-13,5^{\prime}\right)$ appears isolated and is disconnected from weaker extended emission. It may be a filament of gas denser than its environment although we cannot rule out that it is a fold in a sheet. At the opposite, the set of bright structures, in the south of the dense core, are likely pure velocity structures: they gradually move to the north as the velocity decreases. They form a space-velocity bridge between the dense core and the ${ }^{13} \mathrm{CO}$ bright elongated structure. For that reason, the latter will be called the dense core tail. The properties of all these structures are discussed in Sect. 4.

\subsection{Shapes of the line profiles}

Figure 8 displays the spectra (over the new mapped area) of the ${ }^{12} \mathrm{CO}$ and ${ }^{13} \mathrm{CO}(1-0)$ lines averaged over $40 \times 40^{\prime \prime}$ boxes. The line shapes are diverse, even in ${ }^{13} \mathrm{CO}$, a property of molecular

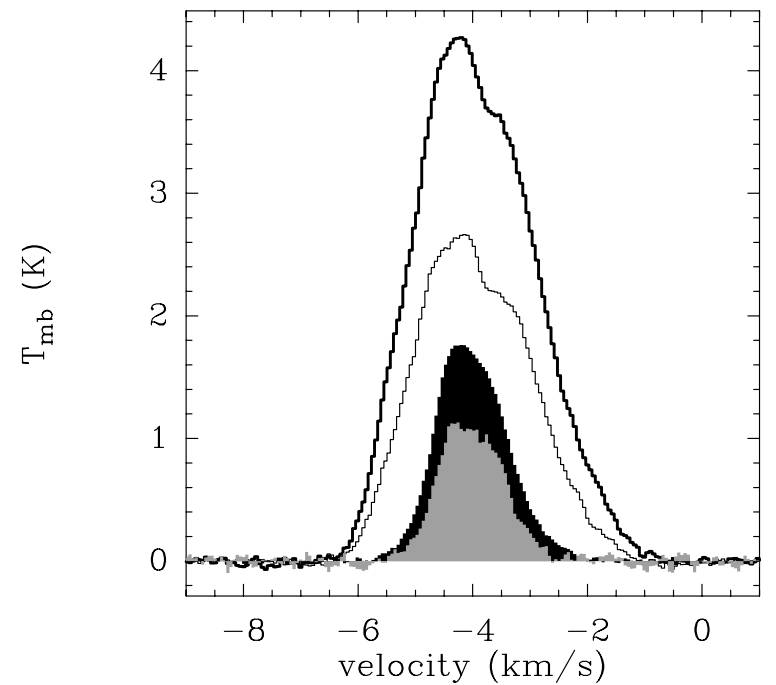

Fig. 5. Averaged spectra, in $T_{\mathrm{mb}}$ scale, over the new maps for each of the four transitions: ${ }^{12} \mathrm{CO}(1-0)$ and $(2-1)$ (full thick and thin lines respectively) and ${ }^{13} \mathrm{CO}(1-0)$ and (2-1) (dark and light filled histograms).

lines which was interpreted as a natural outcome of line formation in turbulent flows by Falgarone et al. (1994), and confirmed later by Monte-Carlo radiative transfer calculations, in three dimensional density and velocity fields from numerical simulations (Padoan et al. 1998). Contrary to the smooth average spectra of Fig. 5, the ${ }^{12} \mathrm{CO}$ lines often present several peaks, some of them having a counterpart in ${ }^{13} \mathrm{CO}$. These spectra maps also show that the far wings in ${ }^{12} \mathrm{CO}$ profiles are present over almost the whole map, with pronounced extrema e.g. at offsets $(-400$, $\left.-50^{\prime \prime}\right)$.

\subsection{Space-velocity maps}

Position-velocity cuts were done over the whole field. A NorthSouth $p-v$ cut performed at constant right ascension $\delta \ell_{\mathrm{II}}=$ $-500^{\prime \prime}$, is shown in Fig. 9. The ${ }^{13} \mathrm{CO}$ cut (left panel) shows that the line is about 4 times broader between $\delta b_{\mathrm{II}}=-220$ and $-20^{\prime \prime}$ than outside and that the transition is very sharp. These offsets correspond to the edges of the ${ }^{13} \mathrm{CO}$ dense core tail at that right ascension (see Fig. 2). Beyond $\delta b_{\mathrm{II}}=-20$, the ${ }^{13} \mathrm{CO} p-v$ cut exhibits a remarkable oscillating pattern centroid in velocity, of wavelength $\approx 200^{\prime \prime}$ that will be discussed in Sect. 6 . This pattern develops in the weak and small North-South structure standing isolated beyond the northern edge of the ${ }^{13} \mathrm{CO}$ integrated map (see Fig. 2), a chance configuration that makes such a weak and peculiar emission unblended with brighter emission and therefore detectable.

The ${ }^{12} \mathrm{CO} p-v$ cut (right panel) illustrates the spatial behavior of the far ${ }^{12} \mathrm{CO}$ wing emission, on the red side at $v>$ $-2 \mathrm{~km} \mathrm{~s}^{-1}$. As expected from its definition, it extends well beyond the dashed line that delineates the ${ }^{13} \mathrm{CO}$ emission level above $3 \mathrm{rms}$ noise level. Its extent experiences large variations over small distances, uncorrelated to variations of the ${ }^{13} \mathrm{CO}$ emission. These sharp variations are at the origin of the large increments of the centroid velocity of the line (i.e. its first velocity moment) measured over small lags. The analysis and statistics of the ${ }^{12} \mathrm{CO}(1-0)$ centroid velocity is the subject of Paper III. 


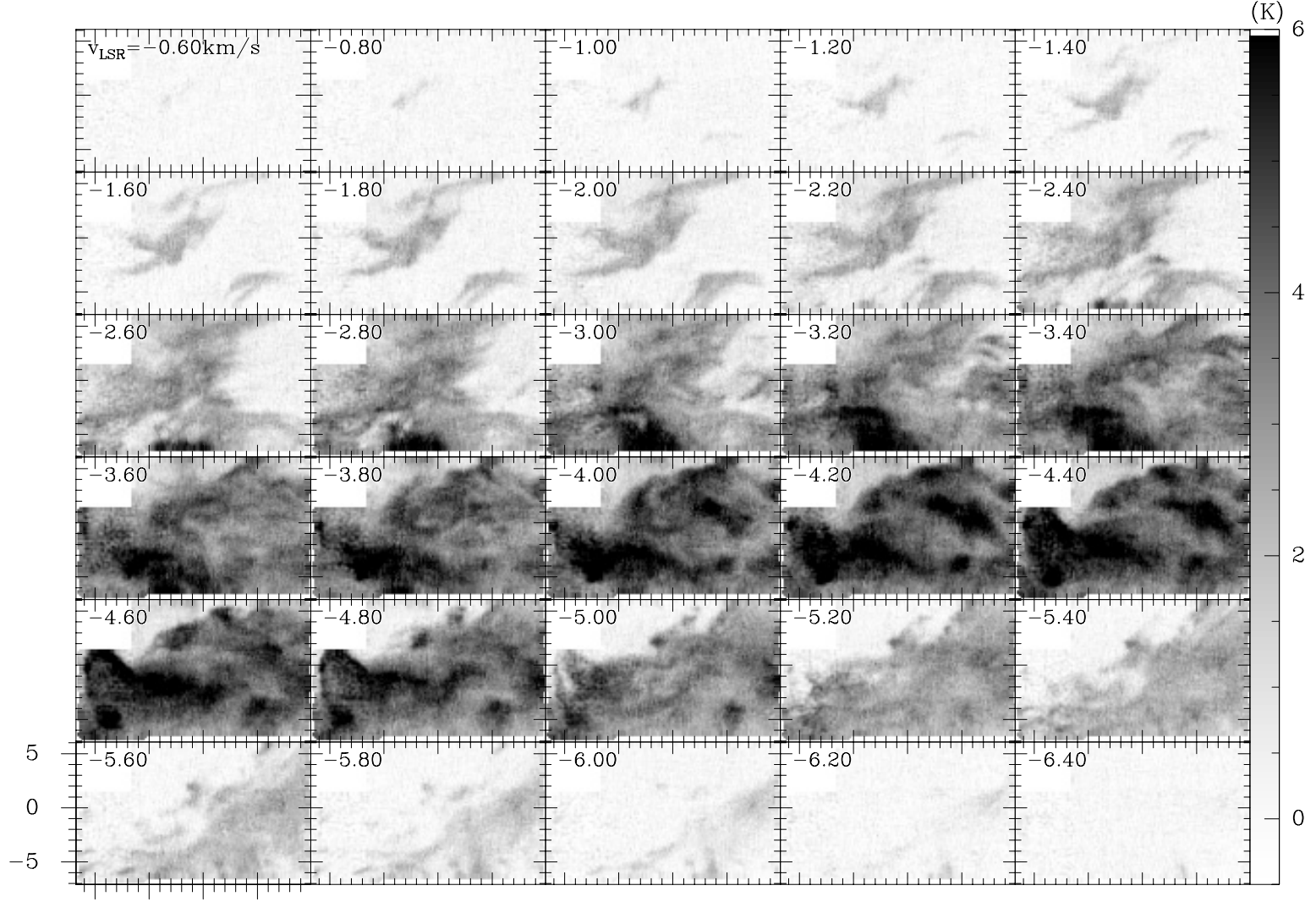

$(\mathrm{K})$

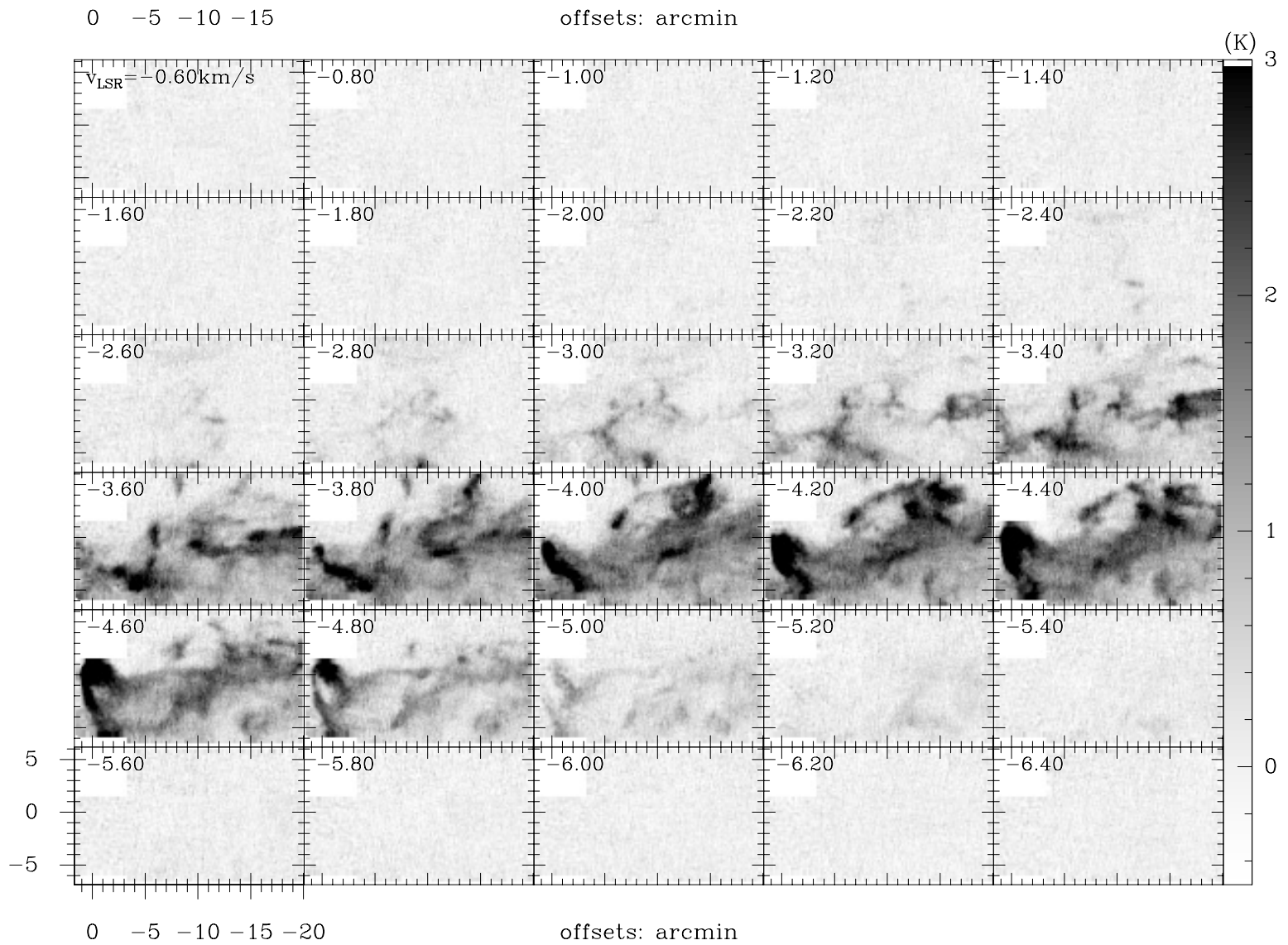

Fig. 6. Channel maps of the whole Polaris field (i.e. including both the new data and those from F98). Top: emission of ${ }^{12} \mathrm{CO}(1-0)$. Offsets are in arcmin. The rms, in each $0.2 \mathrm{~km} \mathrm{~s}^{-1}$ velocity channel, is $0.22 \mathrm{~K}$. Bottom: ${ }^{13} \mathrm{CO}(1-0)$ emission. The rms, in each $0.2 \mathrm{~km} \mathrm{~s}{ }^{-1}$ velocity channel, is $0.14 \mathrm{~K}$. 
$10^{-1}$

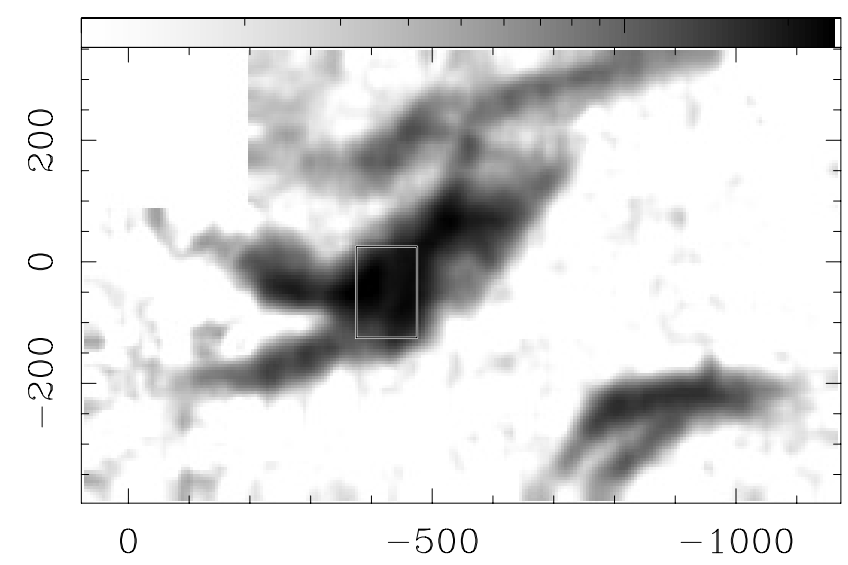

0

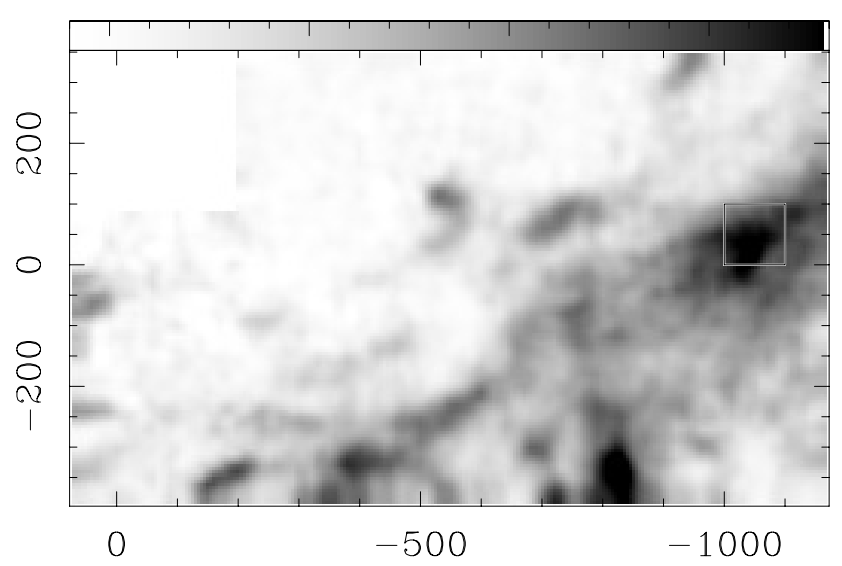

Fig. 7. ${ }^{12} \mathrm{CO}(1-0)$ emission integrated in two velocity intervals where there is no ${ }^{13} \mathrm{CO}$ detected emission: $[-2:-0.5]($ left $)$ and $[-6.5:-5.5] \mathrm{km} \mathrm{s}{ }^{-1}$ (right). Offsets are in arcsec. Grey scale in $\mathrm{K} \mathrm{km} \mathrm{s}^{-1}\left(T_{\mathrm{mb}}\right.$ scale). The boxes are those discussed in Sect. 5.3.

\section{Analysis of the space-velocity structure of the ${ }^{12} \mathrm{CO}$-wing and ${ }^{13} \mathrm{CO}$ emissions}

\subsection{Decomposition algorithm}

To quantitatively characterize the structure seen in the far wings of the ${ }^{12} \mathrm{CO}$ line (Figs. 6 and 7 ), and that seen in the ${ }^{13} \mathrm{CO}$ line (Fig. 6), we have tested two well-known structure analysis algorithms, namely CLUMPFIND (Williams et al. 1994) and GAUSSCLUMP (Stutzki \& Güsten 1990). Both algorithms work on three dimensional data cubes, with the aim of isolating entities along the three directions ( $x, y$ and the line-of-sight velocity). The first algorithm draws iso-contours of intensity and closed 3D-contours are used to define isolate entities. This algorithm therefore makes no assumption about the morphology of the entities nor about its lineshape, and has one key parameter, the spacing between the contours, which was set to $2 \sigma$. The second algorithm searches for Gaussians along the three axes. It first locates the maximum of the intensity and fits a three-dimensional Gaussian, then removes it and iterates on the residuals. A total of 11 input parameters is required. The first method, CLUMPFIND, is unable to remove any large scale component. On the contrary, the GAUSSCLUMP is, by design, biased toward Gaussian-shaped entities. The choice of one or the other thus depends on the type of emission to be analyzed. We have checked the ability of both algorithms to recover artificial filamentary structures. Both algorithms are biased toward the decomposition of large structures into multiple smaller entities. It turned out that the tendency to split large structures into smaller ones was less pronounced with GAUSSCLUMP than with CLUMPFIND, because it is possible to allow the algorithm to fit large Gaussians. We thus decided to use GAUSSCLUMP.

\subsection{Coherent structures in space and velocity}

The algorithm was applied to the full ${ }^{13} \mathrm{CO}$ and ${ }^{12} \mathrm{CO}(1-0)$ data cubes and stopped when $90 \%$ of the total flux was recovered. For each isotopologue, histograms of the aspect ratio and minor axes (deconvolved from telescope beamwidth), velocity $F W H M$ (deconvolved from channel width) and position angle (measured positively from North to East) of the identified Gaussians are shown. As said above, GAUSSCLUMP tends to create a large number of unphysical small scale structures because the Gaussian shape is imposed: only those structures with a peak temperature greater than $3 \sigma$ ( $\sigma$ is respectively 0.30 and $0.20 \mathrm{~K}$ for the ${ }^{12} \mathrm{CO}$ and ${ }^{13} \mathrm{CO}(1-0)$ lines, resampled to coarser velocity resolutions of 0.10 and $0.08 \mathrm{~km} \mathrm{~s}^{-1}$ ) were kept in building the dark-grey histograms. The light-grey histograms are built by further selecting structures with a deconvolved minor axis $>10^{\prime \prime}$.

The results are shown in Fig. 10 for the red ${ }^{12} \mathrm{CO}$ wing (left panel) and ${ }^{13} \mathrm{CO}(1-0)$ (right panel) emission. For the ${ }^{12} \mathrm{CO}$ red wing, structures with a center velocity $v<-3 \mathrm{~km} \mathrm{~s}^{-1}$ were rejected. This limit differs from that mentioned earlier for which the wings are defined according to the ${ }^{12} \mathrm{CO} /{ }^{13} \mathrm{CO}$ line ratio of the average spectra and channel maps. It is the value that allows to best recover the emission seen in Fig. 7 (80\% of the observed wing emission, left panel). Note however that "wing" material may exist in the core velocity range where it is blended with the rest of the gas. GAUSSCLUMP here is used to determine properties of the wing material where we are able to distinguish it in space and velocity from the rest of the gas. We checked that the red wing characteristics remain unchanged when rejecting all structures centered at $v<-2 \mathrm{~km} \mathrm{~s}^{-1}$ instead of $-3 \mathrm{~km} \mathrm{~s}^{-1}$, with obviously a loser statistics and only $50 \%$ of the observed wing emission recovered. The flux in the red wing decomposed by GAUSSCLUMP is mostly ( $>90 \%)$ attributed to resolved structures with minor axis $>10^{\prime \prime}$ and accounts for $20 \%$ of the total observed ${ }^{12} \mathrm{CO}(1-0)$ flux. Most of the decomposed ${ }^{13} \mathrm{CO}$ emission is due to resolved structures ( $85 \%$ of the total ${ }^{13} \mathrm{CO}$ flux).

One of the most interesting result of the GAUSSCLUMP decomposition is the emergence of a characteristic resolved size for the clump minor axis, in both tracers. The ${ }^{12} \mathrm{CO}$ structures have a most probable minor axis of $40 \pm 20$ arcsec or 0.03 pc, larger than that of the ${ }^{13} \mathrm{CO}$ structures which is $20 \pm 10^{\prime \prime}$ or $0.015 \mathrm{pc}$. The most probable velocity widths of the resolved structures are $0.35 \pm 0.15$ and $0.20 \pm 0.10 \mathrm{~km} \mathrm{~s}^{-1}$ in the ${ }^{12} \mathrm{CO}$ and ${ }^{13} \mathrm{CO}$ lines respectively.

The histograms also show that resolved structures are in general elongated with an aspect ratio $2.5 \pm 1$. The exact values of aspect ratios obtained by GAUSSCLUMP are likely artifacts of the procedure that cannot extract bent structures. These elongated structures have a preferred orientation with most probable position angles $\mathrm{PA}=108 \pm 25^{\circ}$ and $\mathrm{PA}=100 \pm 40^{\circ}$ for the ${ }^{12} \mathrm{CO}$ and ${ }^{13} \mathrm{CO}$ respectively.

A third significant result of the GAUSSCLUMP decomposition is the existence of long chains of clumps that form coherent structures of aspect ratio up to 20 (see Fig. 11), with longitudinal velocity variations that increase monotonously with space 

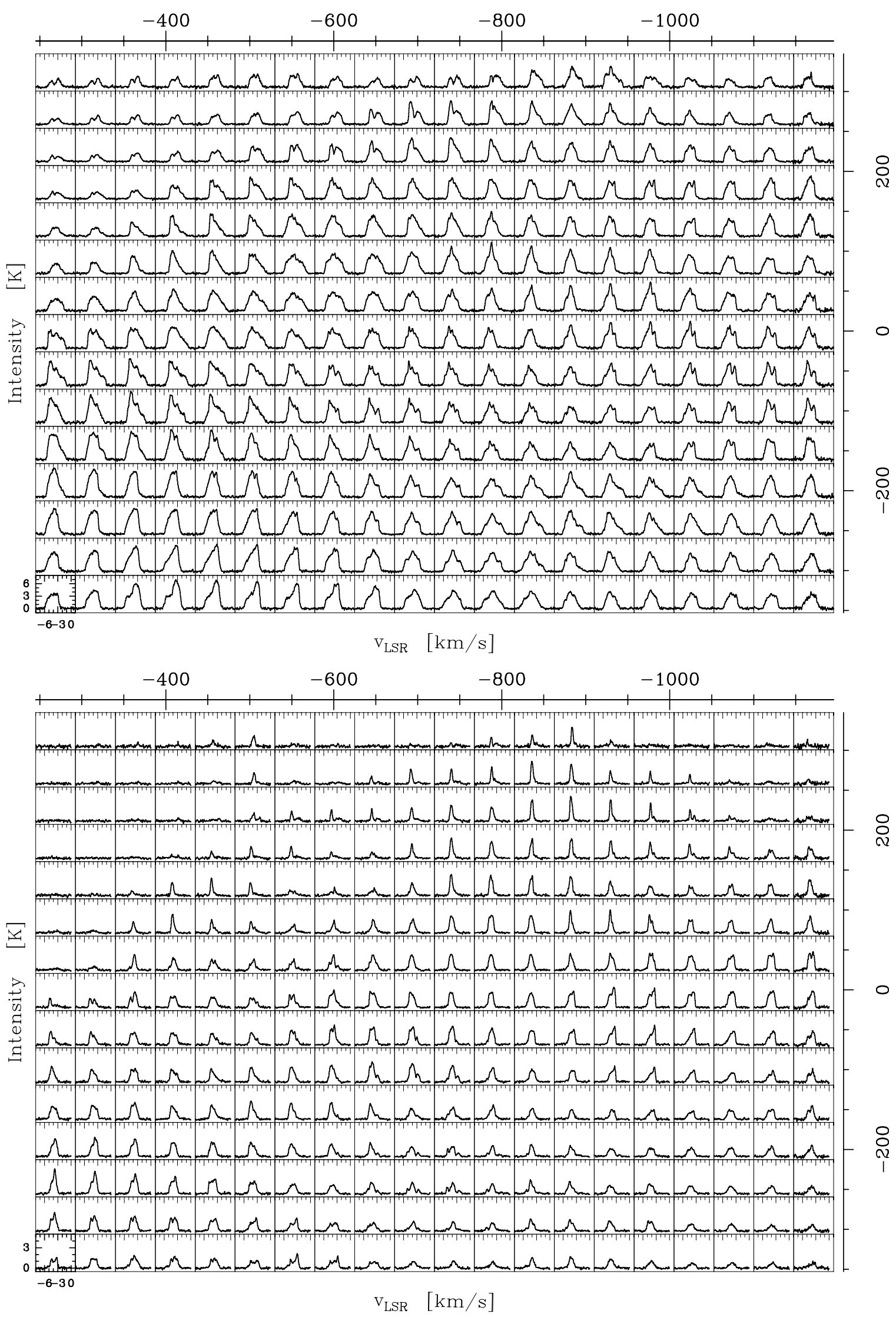

Fig. 8. Spectra maps of ${ }^{12} \mathrm{CO}(1-0)$ (top) and ${ }^{13} \mathrm{CO}(1-0)$ (bottom), in the new data (spectra closer to the dense core are visible in F98), averaged over small regions of $\approx 40 \times 40^{\prime \prime}\left(\approx 20\right.$ spectra). $X$-axis is LSR velocity in $\mathrm{km} \mathrm{s}^{-1}$. The temperature scale is $T_{\mathrm{mb}}$. Offsets (in ") are indicated on the top and the right scales of the plot. 

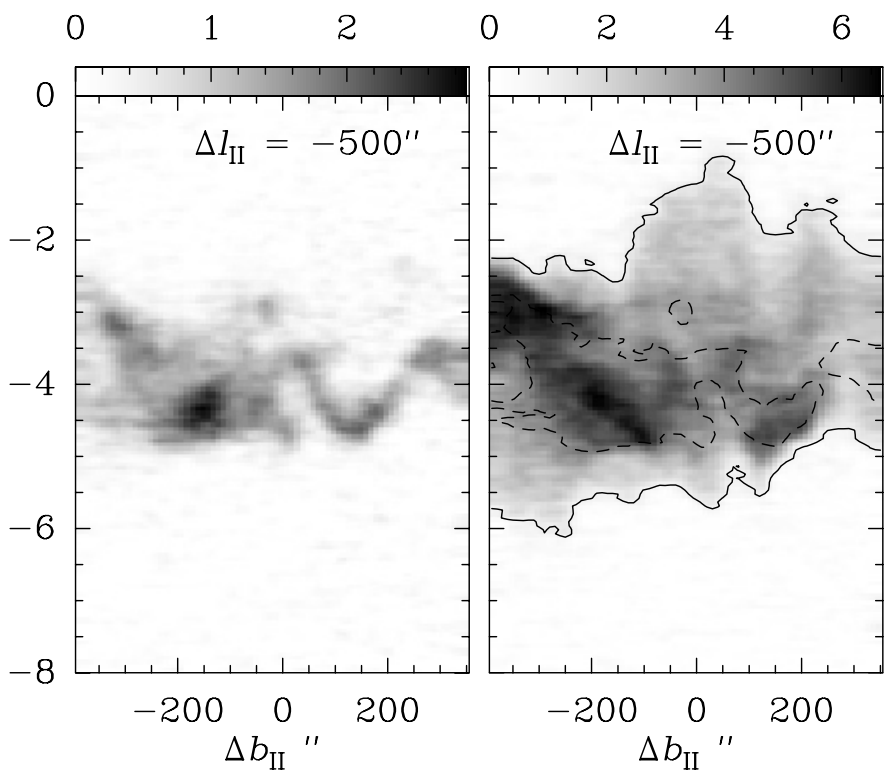

Fig. 9. Position-velocity cuts at $\delta \ell_{\mathrm{II}}=-500^{\prime \prime}$. Left is ${ }^{13} \mathrm{CO}(1-0)$. Right is ${ }^{12} \mathrm{CO}(1-0)$. The dashed contour delineates the structure from the ${ }^{13} \mathrm{CO}$ cut at a level of $1 \mathrm{~K}$. The thick contour shows the $1 \mathrm{~K}$ level of the ${ }^{12} \mathrm{CO}$ emission.

within the error bars (about $0.1 \mathrm{~km} \mathrm{~s}^{-1}$ ) and gradients ranging from 2 to $9 \mathrm{~km} \mathrm{~s}^{-1} \mathrm{pc}^{-1}$. Such long and thin structures, almost crossing the entire field mapped would not have been detected in a smaller map, because their spatial and velocity coherence would have escaped recognition.

\section{Gas properties}

\subsection{Isotopic and line ratios}

The averaged spectra of Fig. 5 provide a parsec scale measurement of the ${ }^{12} \mathrm{CO}$ to ${ }^{13} \mathrm{CO}$ line ratio in the field:

$\left\langle R_{10}\right\rangle=\frac{T_{\text {peak }}\left[{ }^{12} \mathrm{CO}(1-0)\right]}{T_{\text {peak }}\left[{ }^{13} \mathrm{CO}(1-0)\right]}=2.7 \pm 0.4$.

Such a low value compared to the range of abundance ratios in the Solar Neighborhood ( $\approx 40-70$ from Langer et al. 1984) is usually ascribed to the ${ }^{12} \mathrm{CO}(1-0)$ line being optically thick. Fig. 12 (top panel) shows the cross-histogram of $T_{\mathrm{mb}}\left[{ }^{12} \mathrm{CO}(1-\right.$ $0)]$ and $T_{\mathrm{mb}}\left[{ }^{13} \mathrm{CO}(1-0)\right]$. In this kind of diagram, the grey scale indicates the density of points. All velocity channels at all positions are kept, so that the symmetric spot centered on $(0,0)$ gives the noise level. It is seen that the average ratio in fact hides at least two regimes: $i$ ) first an increase of the ${ }^{12} \mathrm{CO}$ temperature, up to $2-3 \mathrm{~K}$, and ii) the saturation of $T_{12}$ while $T_{13}$ continues to increase. The points in regime $i$ ) correspond to the far ${ }^{12} \mathrm{CO}$ line wings discussed in Sect. 3.2. To emphasize properties of regime $i)$, we built the cross-histogram of the ${ }^{12} \mathrm{CO}(1-0)$ against ${ }^{13} \mathrm{CO}(1-0)$ emissions integrated in the blue and red wings (bottom left panel in Fig. 12). While the ${ }^{13} \mathrm{CO}$ is not detected above the noise level, the $W\left({ }^{12} \mathrm{CO}\right)$ increases up to $3 \mathrm{~K} \mathrm{~km} \mathrm{~s}^{-1}$, and linear regression through all data points above a $2 \sigma$ detection threshold gives $W\left({ }^{12} \mathrm{CO}\right) \approx 35 \times W\left({ }^{13} \mathrm{CO}\right)$. This linear increase is characteristic of an optically thin line.

The ratio of the main-beam temperatures in the (2-1) to (1-0) lines has been determined from the corresponding crosshistograms (see Fig. 12). For ${ }^{12} \mathrm{CO}$, it is constant over the whole temperature range, and direct and reverse linear regressions give slopes $R_{12}=0.60$ and 0.80 respectively. These values bracket that, $R_{12}=0.65 \pm 0.15$, found by F98 in the small field around the dense core. For the ${ }^{13} \mathrm{CO}$ line ratio, $R_{13}=0.80 \pm 0.30$ is found when filtering out the points with temperatures less than $2 \sigma$ (dashed lines).

\subsection{Properties of the gas traced by ${ }^{13} \mathrm{CO}(1-0)$ : the dense core tail and its substructures}

The ${ }^{13} \mathrm{CO}$ emission is concentrated in the elongated dense core tail and the dense core itself. The average observed characteristics of the tail and its substructures identified with GAUSSCLUMP are listed in Table 3. The error bars on the line temperatures are the $F W H M / 2.35$ of the histograms built with the points within the dashed polygon of Fig. 2 for the dense core tail, and with the output of GAUSSCLUMP for its substructures. Half-power thickness and linewidth of the substructures are derived from the GAUSSCLUMP analysis (see Sect. 4).

The physical conditions are derived under the large-velocity gradient (LVG) assumptions ${ }^{5}$, with constraints provided by the intensities of the ${ }^{12} \mathrm{CO}(1-0),{ }^{13} \mathrm{CO}(1-0)$ and $(2-1)$ lines. Outputs of the LVG calculations include the kinetic temperature, the density of the colliders $\left(\mathrm{H}_{2}\right)$, the center line opacity and the column density per unit velocity interval $N_{\mathrm{CO}} / \Delta v$. The ${ }^{12} \mathrm{CO}(1-$ $0),{ }^{13} \mathrm{CO}(1-0)$ and $(2-1)$ intensities lead to the solutions $n_{\mathrm{H}_{2}}=$ $3000 \pm 1500 \mathrm{~cm}^{-3}, N_{13} \mathrm{CO} / \Delta v=1.5 \pm 0.5 \times 10^{15} \mathrm{~cm}^{-2}\left(\mathrm{~km} \mathrm{~s}^{-1}\right)^{-1}$ and a kinetic temperature range $T_{\text {kin }}=8-9 \mathrm{~K}$ (see Appendix A Fig. A.1). The dense core and its substructures have the same line intensities (within the error bars) and only their velocity width differ by a factor 8 . Their $\mathrm{H}_{2}$ density and $N_{{ }^{13} \mathrm{CO}} / \Delta v$ are therefore the same, while their column density are in the ratio 8:1 (see Table 3).

To proceed further, we use two complementary constraints on the ${ }^{13} \mathrm{CO}$ abundance relative to $\mathrm{H}_{2}, X_{13}=\left({ }^{13} \mathrm{CO} / \mathrm{H}_{2}\right)$. An upper limit is provided by recent measures of carbon abundances on diffuse to translucent lines of sight by Sofia et al. (2004), $(\mathrm{C} / \mathrm{H})<1.6 \pm 0.2 \times 10^{-4}$, that translates into $X_{13}=$ $X_{12} / 70<4.6 \times 10^{-6}$, in molecular gas where locally all hydrogen is in molecular form and the isotopic ratio $\left({ }^{12} \mathrm{CO} /{ }^{13} \mathrm{CO}\right)=70$. A lower limit is provided by the condition that the total column density of hydrogen nuclei in the tail does not exceed the total column density of the field in its most opaque parts, $N_{\mathrm{H} \text {,tail }}<N_{\mathrm{H}}=1.5 \times 10^{21} \mathrm{~cm}^{-2}$, or $X_{13}>3.2 \times 10^{-6}$. The upper and lower limits on $X_{13}$ are therefore very close and in the following we adopt $X_{13}=3.2 \times 10^{-6}$. The associated $\mathrm{H}_{2}$ density derived from the size of the core tail is $n_{\mathrm{H}_{2}}=N\left({ }^{13} \mathrm{CO}\right) /\left(X_{13} D\right)=$ $1800 \pm 600 \mathrm{~cm}^{-3}$, in the range of the LVG solutions, and we adopt in the following $n_{\mathrm{H}_{2}}=2000 \mathrm{~cm}^{-3}$. The column density of the substructures is thus $N_{\mathrm{H}_{2} \text {,sub }}=10^{20} \mathrm{~cm}^{-2}$.

The gas seen in ${ }^{13} \mathrm{CO}$ is therefore dense with $n_{\mathrm{H}_{2}} \approx$ $2000 \mathrm{~cm}^{-3}$ and cold with $T_{\text {kin }}=8-9 \mathrm{~K}$. The gas density, on average, is the same for the dense core tail and its substructures, so these substructures are pure velocity structures: they fill the whole volume of their parent structure and they are identified by GAUSSCLUMP in velocity space only. If further substructure exists in the beam, an analysis similar to that followed in F98 and inspired by the approach developed by Martin et al. (1984) would lead to denser gas solutions.

5 The code was written by M. Pérault. The $\mathrm{CO}-\mathrm{H}_{2}$ cross-sections are taken from Flower (2001), for kinetic temperatures ranging from 5 to $400 \mathrm{~K}$, and from McKee et al. (1982) for $T_{\text {kin }}=500-2000 \mathrm{~K}$. Linear interpolation was used for untabulated temperatures (no extrapolation). 

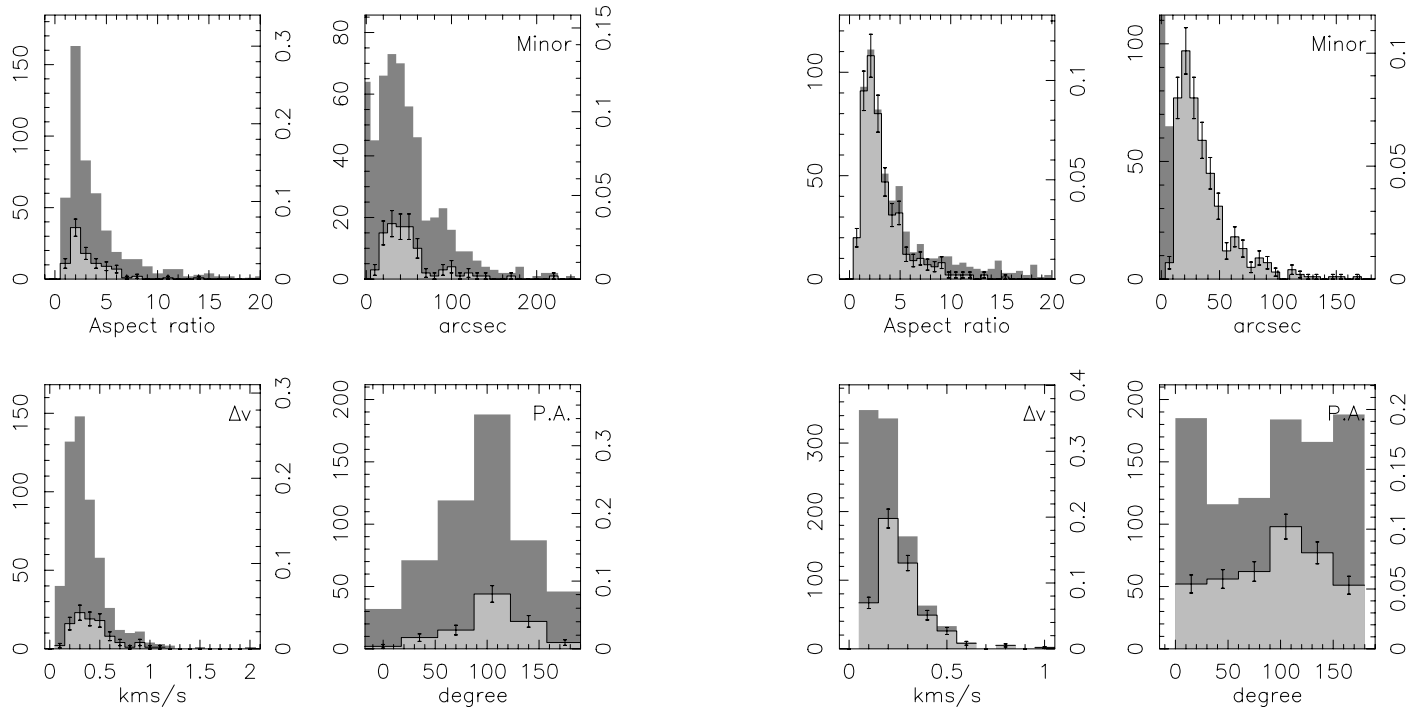

Fig. 10. Histograms from the GAUSSCLUMP decomposition of the ${ }^{12} \mathrm{CO}(1-0)$ red wing (left panels) and ${ }^{13} \mathrm{CO}$ (right panels) emissions. Minor axis and velocity $F W H M$ are deconvolved from the spatial and spectral resolutions. In each panel, the right $Y$-scale gives the histogram normalized to unity. The error bars are statistical errors $(\sqrt{N})$. Dark-grey histograms contain all structures with $T>3 \sigma$, and light-grey ones contain only structures with a minor axis $>10^{\prime \prime}$. For the ${ }^{12} \mathrm{CO}$, only those structures centered in the range [-3:-0.5] $\mathrm{km} \mathrm{s}^{-1}$ were kept in the light-grey histograms (see text).
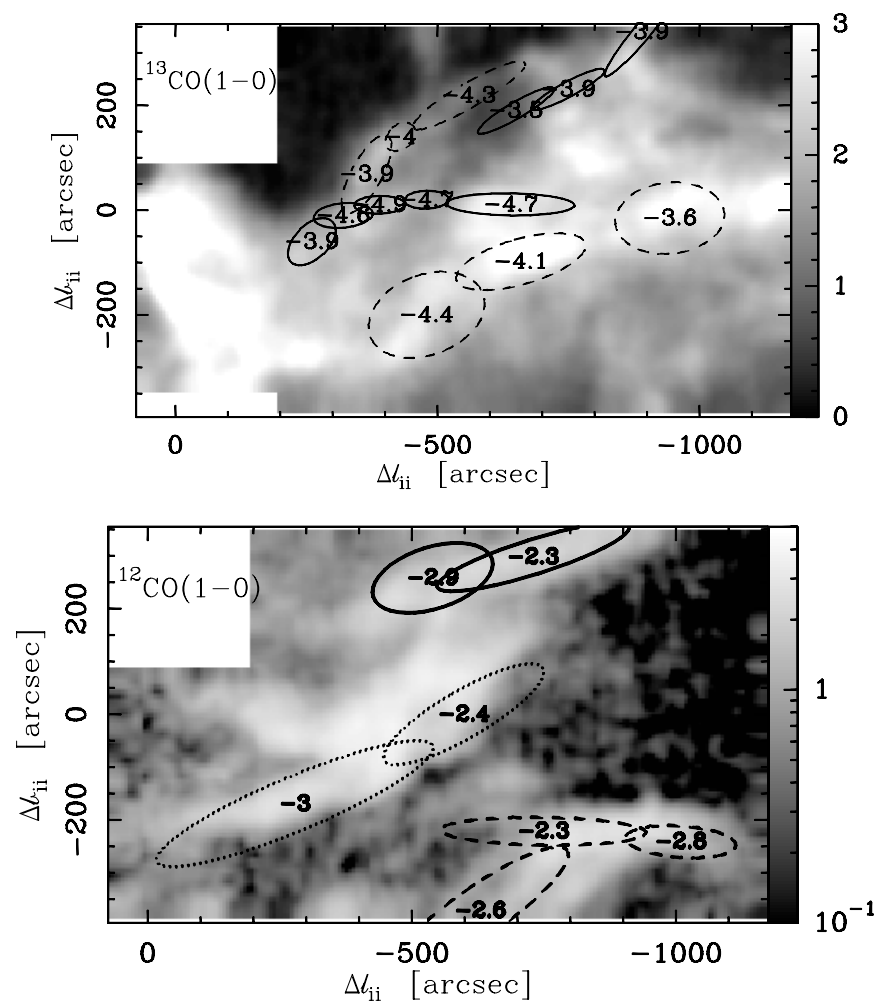

Fig. 11. Top: Gaussians structures, from the GAUSSCLUMP decomposition of the ${ }^{13} \mathrm{CO}$ emission, forming coherent ensembles, are displayed, with their central velocities. Bottom: same as above for the ${ }^{12} \mathrm{CO}$ red wing emission. Grey-scales are $\mathrm{K} \mathrm{km} \mathrm{s}^{-1}$.

\subsection{Gas in the far ${ }^{12} \mathrm{CO}$ line wings}

To emphasize the observed properties of the gas detected only in the far wings of the ${ }^{12} \mathrm{CO}$ lines (see Sect. 3), we have integrated the ${ }^{12} \mathrm{CO}$ emission over two boxes in the field where the blue and red wings are the most prominent (see Fig. 7). The integrated spectra are displayed in Fig. 13 where each of the far
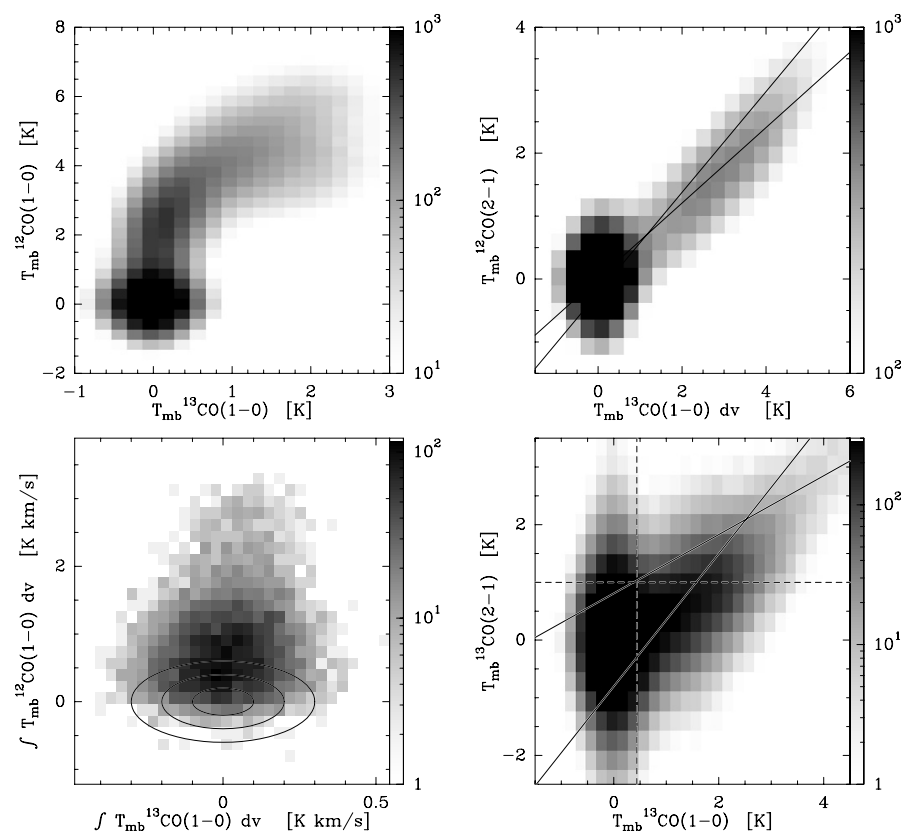

Fig. 12. Left panels: Top: cross histogram of $T_{\mathrm{mb}}$ of ${ }^{12} \mathrm{CO}(1-0)$ versus ${ }^{13} \mathrm{CO}(1-0)$, for all velocity channels. Bottom: cross histogram of the ${ }^{12} \mathrm{CO}(1-0)$ vs. ${ }^{13} \mathrm{CO}(1-0)$ integrated main beam-intensities, computed only in the ${ }^{12} \mathrm{CO}$-wings (see Sect. 5.1). The ellipses indicate the 1, 2 and $3 \sigma$ levels. Right panels: cross histograms of (2-1) against (1-0) corrected main beam temperatures, for each isotopologue. Top: ${ }^{12} \mathrm{CO}$. Least square linear fits have slopes 0.60 and 0.80 . Bottom: same as before, for the ${ }^{13} \mathrm{CO}$ with slopes 0.5 and 1.15 , determined with the values above the dashed lines.

wing domain is shaded. From Table 4, the far wings ${ }^{12} \mathrm{CO}(1-$ 0 ) peak line intensities are similar, $T_{12} \approx 2.3 \mathrm{~K}$ and they are characterized by a large line ratio $R_{10} \geq 30$. Their CO column densities and $\mathrm{H}_{2}$ densities are derived in the LVG formalism, with $T_{12}=1.9-2.6 \mathrm{~K}$ and the additional constraint from the well defined line ratio $R_{12}=0.70 \pm 0.10$. 
Table 3. Properties of the dense core tail (averaged within the dashed polygon of Fig. 2) and its substructures as extracted by GAUSSCLUMP from the ${ }^{13} \mathrm{CO}$ emission: main-beam peak temperatures, half-power thickness $(D)$, velocity width $(\Delta v) . \mu$ is the mass per unit length assuming (see Eq. (2)), and $\mu_{\mathrm{vir}}$ is the virial mass assuming a cylindrical symmetry (see Sect. 6.1, Eq. (3)). We indicate in Col. 3 the origin of the values used ( $\mathrm{Gcl}$ stands for GAUSSCLUMP for the substructures).

\begin{tabular}{llccc}
\hline \hline & & & Tail & Substructures \\
\hline$T_{12}(1-0)$ & {$[\mathrm{K}]$} & obs & $5.0 \pm 0.7$ & $4.4 \pm 0.4$ \\
$T_{13}(1-0)$ & {$[\mathrm{K}]$} & obs & $1.9 \pm 0.3$ & $1.4 \pm 0.4$ \\
$T_{12}(2-1)$ & {$[\mathrm{K}]$} & obs & $3.5 \pm 1.0$ & $2.7 \pm 0.6$ \\
$T_{13}(2-1)$ & {$[\mathrm{K}]$} & obs & $0.9 \pm 0.2$ & $0.8 \pm 0.2$ \\
$D$ & {$[\mathrm{pc}]$} & Gcl & $0.13 \pm 0.02$ & $0.015 \pm 0.007$ \\
$\Delta v$ & {$\left[\mathrm{~km} \mathrm{~s}^{-1}\right]$} & Gcl & 1.6 & 0.2 \\
$N\left({ }^{13} \mathrm{CO}\right)$ & {$\left[10^{15} \mathrm{~cm}^{-2}\right]$} & LVG & $2.4 \pm 0.8$ & $0.3 \pm 0.1$ \\
$X_{13}$ & {$\left[\times 10^{-5}\right]$} & LVG & 0.32 & 0.32 \\
$\mu$ & {$\left[M_{\odot} \mathrm{pc}^{-1}\right]$} & & 2.2 & 0.03 \\
$\mu_{\text {vir }}$ & {$\left[M_{\odot} \mathrm{pc}^{-1}\right]$} & & 215 & 3.5 \\
$\mu / \mu_{\text {vir }}$ & & & 0.01 & 0.01 \\
\hline
\end{tabular}

Table 4. Observational properties in the far ${ }^{12} \mathrm{CO}$ line wings determined in the two boxes of Fig. 7. $\Delta v_{\text {box }}$ is the equivalent width.

\begin{tabular}{lccccc}
\hline \hline Wing & $\begin{array}{c}T_{12}(1-0) \\
{[\mathrm{K}]}\end{array}$ & $\begin{array}{c}T_{13}(1-0) \\
{[\mathrm{K}]}\end{array}$ & $\begin{array}{c}\int T_{12} \mathrm{~d} v \\
{\left[\mathrm{~K} \mathrm{~km} \mathrm{~s}^{-1}\right]}\end{array}$ & $R_{10}$ & $\begin{array}{c}\Delta v_{\text {box }} \\
{\left[\mathrm{km} \mathrm{s}^{-1}\right]}\end{array}$ \\
\hline red & $2.25 \pm 0.35$ & $\leq 0.07$ & $2.10 \pm 0.02$ & $\geq 30$ & 0.8 \\
blue & $2.30 \pm 0.20$ & $\leq 0.07$ & $1.50 \pm 0.02$ & $\geq 30$ & 0.5 \\
\hline
\end{tabular}

Solutions have been searched for $T_{\text {kin }}=5-2000 \mathrm{~K}$. Results for $T_{\text {kin }}=10 \mathrm{~K}$ and $100 \mathrm{~K}$ are shown in Fig. A.2 (Appendix A). Figure 13 (right panel) shows the resulting set of solutions for kinetic temperatures ranging from 7 to $400 \mathrm{~K}$, the upper limit being provided by the CO linewidths, $\Delta v_{\text {box }} \leq 0.8 \mathrm{~km} \mathrm{~s}^{-1}$, assuming they are purely thermal.

The coldest solutions can securely be eliminated because they would have $\mathrm{H}_{2}$ column densities too large for this translucent field. The GAUSSCLUMP analysis showed that the far wing ${ }^{12} \mathrm{CO}$ emission is structured in space and velocity. We therefore assume that this emission is distributed into filaments of linewidth $0.35 \mathrm{~km} \mathrm{~s}^{-1}$ and thickness $0.03 \mathrm{pc}$ (Sect. 4.2). The comparison of the linewidths of the boxes $\left(\Delta v_{\text {box }}=0.5\right.$ and $0.8 \mathrm{~km} \mathrm{~s}^{-1}$ ) in which the LVG analysis is conducted with the filament linewidth suggests that each box contains more than one filament, as suggested by Fig. 7. The total $\mathrm{H}_{2}$ column density in each box cannot exceed $\left(\Delta v_{\text {box }} / \Delta v_{\text {tot }}\right) N_{\mathrm{H}_{2}}=1.5-2.4 \times 10^{20} \mathrm{~cm}^{-2}$, for the blue and red wings respectively, where we have used a very conservative scaling of the column density with velocity coverage $\left(\Delta v_{\text {tot }}=2.5 \mathrm{~km} \mathrm{~s}^{-1}\right)$. This gives upper limits on the density, $n_{\mathrm{H}_{2}}=800$ and $1300 \mathrm{~cm}^{-3}$, with associated kinetic temperatures $T_{\text {kin }}>30$ and $20 \mathrm{~K}$. The ${ }^{12} \mathrm{CO}(1-0)$ center line opacity is lower than 0.2 (see Fig. 13). On average, the gas properties in the wings are $T_{\text {kin }}>25 \mathrm{~K}$ and $n_{\mathrm{H}_{2}}<1000 \mathrm{~cm}^{-3}$, and the abundance is $X_{12}>\left(N_{\mathrm{CO}} / \Delta v\right) \Delta v /\left(n_{\mathrm{H}_{2}} D\right)=6 \times 10^{-6}$ (see Table 5).

The gas responsible for the far wings ${ }^{12} \mathrm{CO}$ emission is warm and dilute, optically thin in ${ }^{12} \mathrm{CO}(1-0)$, and has a well-defined $\mathrm{CO}$ column density per unit velocity, $N_{\mathrm{CO}} / \Delta v=1.7 \pm 0.1 \times$ $10^{15} \mathrm{~cm}^{-2}\left(\mathrm{~km} \mathrm{~s}^{-1}\right)^{-1}$. The thermal pressure of all the solutions varies from $P_{\mathrm{th}} / k=3$ to $8 \times 10^{4} \mathrm{~K} \mathrm{~cm}^{-3}$. It is found in thin $(0.03 \mathrm{pc})$ and elongated (up to $0.5 \mathrm{pc})$ structures covering a large fraction of the field area $(\approx 60 \%$, see below). The gas properties are summarized in Table 5. It is noteworthy that a similar domain of gas densities and temperatures $\left(T_{\text {kin }}>25 \mathrm{~K}\right.$ and $n_{\mathrm{H}_{2}}<10^{3} \mathrm{~cm}^{-3}$ ) has been obtained for gas emitting in ${ }^{12} \mathrm{CO}$ far
Table 5. Properties of the thin ${ }^{12} \mathrm{CO}$-filaments from the LVG analysis (see Sect. 5.3 and Table 4) and the GAUSSCLUMP analysis, applied to the coldest and warmest solutions. $N_{\mathrm{H}_{2}}=n_{\mathrm{H}_{2}} D, X_{12}=N\left({ }^{12} \mathrm{CO}\right) / N_{\mathrm{H}_{2}}$. Masses per unit length follow from Eqs. (2) and 4, where upper limits are $1 \sigma$. We indicate in Column 3 the origin of the values used $(\mathrm{Gcl}$ stands for GAUSSCLUMP).

\begin{tabular}{|c|c|c|c|c|}
\hline Parameter & Unit & & \multicolumn{2}{|c|}{ "Value } \\
\hline$N_{\mathrm{CO}} / \Delta v$ & {$\left[\mathrm{~cm}^{-2} / \mathrm{km} \mathrm{s}^{-1}\right]$} & $\overline{L V G}$ & \multicolumn{2}{|c|}{$1.7 \pm 0.1 \times 10^{15}$} \\
\hline$\tau_{12}$ & & LVG & \multicolumn{2}{|c|}{$<0.2$} \\
\hline$D$ & [pc] & $\mathrm{Gcl}$ & \multicolumn{2}{|c|}{$0.030 \pm 0.015$} \\
\hline & {$\left[\mathrm{km} \mathrm{s}^{-1}\right]$} & $\mathrm{Gcl}$ & \multicolumn{2}{|c|}{$0.35 \pm 0.15$} \\
\hline$N\left({ }^{12} \mathrm{CO}\right)$ & {$\left[\mathrm{cm}^{-2}\right]$} & & \multicolumn{2}{|c|}{$0.6 \pm 0.3 \times 10^{15}$} \\
\hline$\mu_{\mathrm{vir}}$ & {$\left[M_{\odot} \mathrm{pc}^{-1}\right]$} & & \multicolumn{2}{|c|}{$10 \pm 8$} \\
\hline$T_{\text {kin }}$ & {$[\mathrm{K}]$} & LVG & $>25$ & $<400$ \\
\hline$n\left(\mathrm{H}_{2}\right)$ & {$\left[\mathrm{cm}^{-3}\right]$} & LVG & $<1000$ & $>200$ \\
\hline$N_{\mathrm{H}_{2}}$ & {$\left[\times 10^{20} \mathrm{~cm}^{-2}\right]$} & & $<1.0 \pm 0.5$ & $>0.2 \pm 0.1$ \\
\hline$X_{12}$ & {$\left[\times 10^{-5}\right]$} & & $>0.6 \pm 0.3$ & $<3.2 \pm 2.0$ \\
\hline$\mu$ & {$\left[M_{\odot} \mathrm{pc}^{-1}\right]$} & & $<0.10$ & $<0.01$ \\
\hline$\mu / \mu_{\mathrm{vir}}$ & & & $<0.01$ & $<0.001$ \\
\hline
\end{tabular}
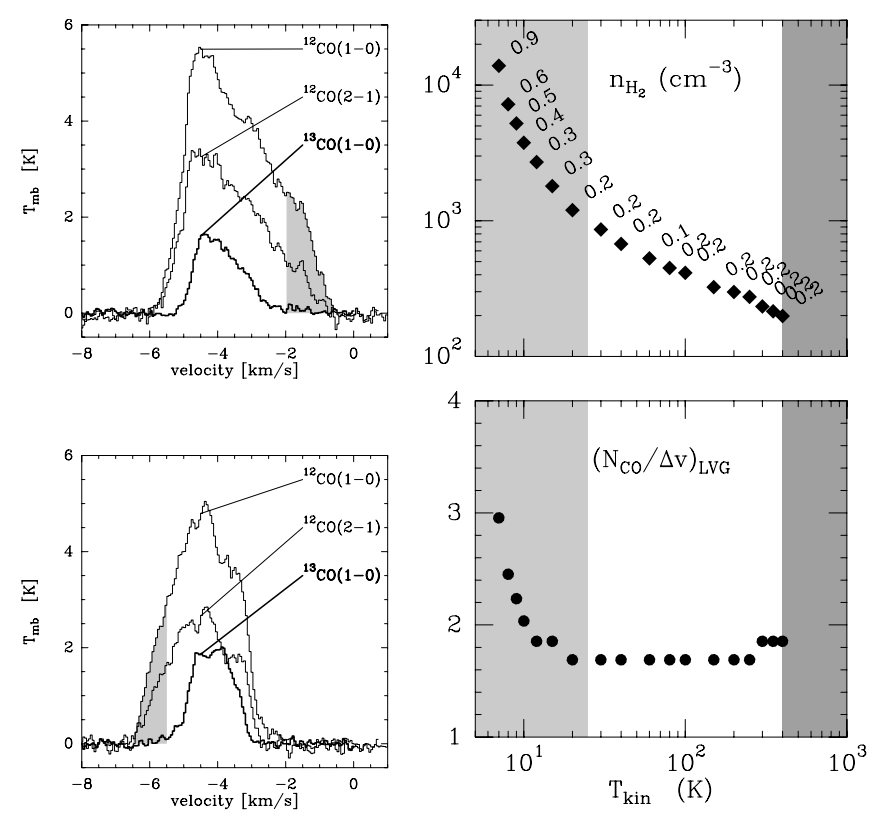

Fig. 13. Average spectra of the ${ }^{12} \mathrm{CO}(1-0)$, (2-1) and ${ }^{13} \mathrm{CO}(1-0)$ lines over the two boxes of Fig. 7 emphasizing the red and blue ${ }^{12} \mathrm{CO}$ wings (top and bottom left resp.). Right panels: $\mathrm{H}_{2}$ density and $\tau_{12}$ (numbers) from the LVG calculations (top, see Fig. A.2 of Appendix A) and the column density per unit velocity interval in units of $10^{15} \mathrm{~cm}^{-2}\left(\mathrm{~km} \mathrm{~s}^{-1}\right)^{-1}$ (bottom). The shaded areas indicate the discarded domains of solutions (see text).

wings in another field by a completely different method based on the comparison of the intensities of the four lowest rotational transition of ${ }^{12} \mathrm{CO}$ (Falgarone \& Phillips 1996). This solution is different from that of F98 where the entire ${ }^{12} \mathrm{CO}$ emission was proposed to arise from unresolved dense clumps with spacevelocity beam filling factors varying across the line profiles. With only two CO transitions, it is difficult to decide whether or not beam dilution is important. However, our present set of warm and dilute solutions is supported by the large $\mathrm{HCO}^{+}$abundances inferred from observations in the direction of one of these filaments (Paper I). 
Table 6. Masses of various structures identified in the field (Eq. (1)). (1): $N_{\mathrm{CO}}$ is derived from the ${ }^{13} \mathrm{CO}$ column density assuming an abundance ratio of 70. (2): from Heithausen (2002). (3): total mass derived from the mean visual extinction.

\begin{tabular}{lccccccr}
\hline \hline Structure & Tracer & $\begin{array}{c}\Sigma_{36} \\
10^{36} \mathrm{~cm}^{-2}\end{array}$ & $\begin{array}{c}X_{-5} \\
10^{-5}\end{array}$ & $\begin{array}{c}N_{\mathrm{CO}} \\
10^{15} \mathrm{~cm}^{-2}\end{array}$ & $\begin{array}{c}N_{21} \\
10^{21} \mathrm{~cm}^{-2}\end{array}$ & $\begin{array}{c}M_{\mathrm{H}} \\
M_{\odot}\end{array}$ & $\%$ \\
\hline Red Wing & ${ }^{12} \mathrm{CO}$ & 1.0 & $>0.6$ & 0.8 & $<0.3$ & $<0.3$ & $<4.9$ \\
Blue Wing & ${ }^{12} \mathrm{CO}$ & 1.7 & $>0.6$ & 0.6 & $<0.2$ & $<0.4$ & $<6.3$ \\
Core Tail $^{(1)}$ & ${ }^{13} \mathrm{CO}$ & 1.4 & 0.3 & 2.3 & $<1.5$ & $<2.6$ & $<39.6$ \\
Dense core $^{(2)}$ & & - & - & - & - & 1.0 & 15.4 \\
Envelope & & - & 1.0 & - & - & $>2.2$ & $>33.8$ \\
Whole field $^{(3)}$ & $A_{\mathrm{V}}$ & 4.2 & - & - & 1.3 & 6.5 & 100.0 \\
\hline
\end{tabular}

\section{Masses and dynamics of the various structures in the field}

\subsection{Gas mass distribution}

The translucent field adjacent to the dense core therefore comprises $i$ ) a dense core tail about $1 \mathrm{pc}$ long, bright in ${ }^{13} \mathrm{CO}$ with marked substructure in velocity space, and $i$ ) gas optically thin in the ${ }^{12} \mathrm{CO}(1-0)$ line, forming elongated structures (called the optically thin ${ }^{12} \mathrm{CO}$-filaments in what follows). Yet, these two components do not account for the whole column density in the field estimated from star counts (Sect. 2). We introduce here what we call the envelope which takes into account the extinction of the field and is neither bright in ${ }^{13} \mathrm{CO}$ (the dense core tail) nor emitting in the ${ }^{12} \mathrm{CO}$ line wings (the optically thin ${ }^{12} \mathrm{CO}$ filaments). The envelope includes gas weak or undetected in ${ }^{13} \mathrm{CO}$, and gas undetected in ${ }^{12} \mathrm{CO}$ (molecular and atomic hydrogen, possibly ionized). The optically thin ${ }^{12} \mathrm{CO}$-filaments are likely embedded in it.

We estimate below the mass of each of these components using their total projected area in the map, $\Sigma$ and their column density of $\mathrm{H}$ nuclei $N_{\mathrm{H}}$ :

$M_{\mathrm{H}}=1.4 \Sigma m_{\mathrm{H}} N_{\mathrm{H}}=1.2 M_{\odot} \Sigma_{36} N_{21}$

where $\Sigma_{36}$ is expressed in $10^{36} \mathrm{~cm}^{-2}$ and $N_{21}$ is the total hydrogen column density $\left(N_{\mathrm{H}}=N_{\mathrm{HI}}+2 N_{\mathrm{H}_{2}}\right)$ in units of $10^{21} \mathrm{~cm}^{-2}$, derived from either the ${ }^{12} \mathrm{CO}$ or ${ }^{13} \mathrm{CO}$ column densities or star counts for the whole field. The total mass of the field $\left(6.5 M_{\odot}\right)$ is inferred from the average extinction (Sect. 2) and the size of the map. The CO column density averaged over the whole map, in each of the two wing velocity intervals, is derived from their $\mathrm{CO}$ integrated emission $\left\langle W_{\mathrm{CO}}\right\rangle_{\text {wing }}$ by $\left\langle N_{\mathrm{CO}}\right\rangle_{\text {wing }}=\left(N_{\mathrm{CO}} / W_{\mathrm{CO}}\right)_{\text {box }} \times\left\langle W_{\mathrm{CO}}\right\rangle_{\text {wing }}$, with $\left\langle W_{\mathrm{CO}}\right\rangle_{\text {wing }}=1.3$ and $1.0 \mathrm{~K} \mathrm{~km} \mathrm{~s}^{-1}$ in the red and blue wing velocity ranges respectively. The mass of the envelope is the difference between the total mass and the sum of all the components (dense core, dense core tail, ${ }^{12} \mathrm{CO}$-filaments).

The results are given in Table 6 . The dense core tail accounts for more than one third of the total mass, and is likely more massive than the dense core itself $\left(1 M_{\odot}\right)$. The optically thin ${ }^{12} \mathrm{CO}-$ filaments account for less than $13 \%$ of the total mass.

\subsection{Comparison of the gas masses to their virial masses}

In this section, we compare the gas mass and the virial mass per unit length of the optically thin ${ }^{12} \mathrm{CO}$-filaments and the dense core tail. The gas mass per unit length writes, in cylindrical geometry,

$\mu=2.8 m_{\mathrm{H}} D N_{\mathrm{H}_{2}}=0.22 M_{\odot} \mathrm{pc}^{-1} D_{0.1} N_{15} / X_{-5}$

where $D_{0.1}$ is the projected thickness of the structure, $N_{15}$ its $\mathrm{CO}$ column density and $X_{-5}=\left(\mathrm{CO} / \mathrm{H}_{2}\right)$ the $\mathrm{CO}$ abundance, respectively in units of $0.1 \mathrm{pc}, 10^{15} \mathrm{~cm}^{-2}$ and $10^{-5}$. The mean mass per $\mathrm{H}$ nuclei is $\tilde{m}=1.4 m_{\mathrm{H}}$ assuming an He abundance of $10 \%$ relative to $\mathrm{H}$. The virial mass per unit length in cylindrical geometry is: (e.g. Fiege \& Pudritz 2000a)

$\mu_{\mathrm{vir}}=\frac{2\left\langle\sigma_{0}^{2}\right\rangle}{G}=\frac{2\left\langle\Delta v_{0}^{2}\right\rangle / 8 \ln (2)}{G}$

where $\sigma_{0}$ is the $1 D$ velocity dispersion of the gas, which is deduced from that of $\mathrm{CO}$ by correcting from the $\mathrm{CO}$ molecular weight (Fuller \& Myers 1992):

$\mu_{\mathrm{vir}}=84 \Delta v_{0}^{2} M_{\odot} \mathrm{pc}^{-1}$

with $\Delta v_{0}$ in $\mathrm{km} \mathrm{s}^{-1}$.

Results for the dense core tail and the optically thin ${ }^{12} \mathrm{CO}-$ filaments are given in Tables 3 and 5: the gas masses are well below the virial masses. As expected, the dense core tail has the same $\mu / \mu_{\text {vir }}$ ratio as its substructures because $\mu / \mu_{\text {vir }} \propto D / \Delta v$ and its internal velocity dispersion approximately scales as $\Delta v \propto D$ (Table 3).

\subsection{The conspicuous oscillating velocity pattern}

As mentioned in Sect. 3.5, the $p-v$ cut performed at constant $\delta \ell_{\text {II }}=-500^{\prime \prime}$ in the ${ }^{13} \mathrm{CO}(1-0)$ emission (Fig. 9, left panel) exhibits a peculiar oscillation of the velocity with position, for $\delta b_{\mathrm{II}}>50^{\prime \prime}$. The wavelength of the oscillation is $\lambda \approx 200^{\prime \prime}=$ $0.15 \mathrm{pc}$. This oscillation is observed in all the $p-v$ cuts with $\delta \ell_{\mathrm{II}}$ in the range [ $\left.-520:-470^{\prime \prime}\right]$, while the integrated emission (as noted in Sect. 3.5) only shows a weak and just resolved straight feature running N-S (Fig. 2). Therefore, the oscillation is only visible through the spatial resolution of the gas velocity variations. A simple interpretation of the oscillation would be that of a gas flowing along an helical structure of diameter $0.015 \mathrm{pc}$.

We have modeled such a flow and produced synthetic data cubes, with resolutions (spectral and spatial) corresponding to our observations. The key parameter is the ratio of the radius $r$ to the wavelength $\lambda$ of the helix (see Appendix B). If the ratio $\lambda / r$ is large enough, the helix is then resolved in its longitudinal direction, using the velocity information to catch its step, while it is not necessarily resolved in its transverse direction. Figure 14 shows a $p-v$ cut across a synthetic helix of wavelength $\lambda=200^{\prime \prime}$ (corresponding to the observed value) with $\lambda / r=20$, i.e. an helix of diameter $20^{\prime \prime}$ or $0.015 \mathrm{pc}$. Taking the convolution with a beam of $22^{\prime \prime}$ into account, $p-v$ cuts at offsets $\delta y \pm 30^{\prime \prime}$ are identical but weaker, as observed.

Helical magnetic fields would naturally produce such a flow since gas, even weakly ionized, preferentially flows along magnetic field lines. We cannot disentangle this motion from that of an helix of matter in solid rotation, uniform over 200". Another 

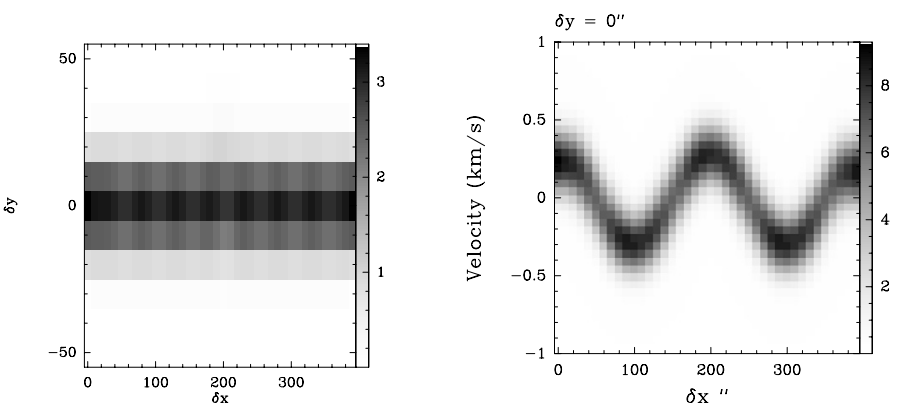

Fig. 14. Gas flowing along an helical structure lying in the plane of the sky. Left: integrated intensity map. Right: $\delta x-v$ cut at constant $\delta y=0^{\prime \prime}$ offset. The wavelength corresponds to the observed one, $\lambda=200^{\prime \prime}$.

scenario would be that of a tilted cylindrical filament experiencing periodic inflows along its main axis. This would generate density enhancements that are not visible in Fig. 2.

\section{Discussion}

\subsection{The optically thin ${ }^{12} \mathrm{CO}$-filaments}

It is interesting to compare the properties of the gas in the optically thin ${ }^{12} \mathrm{CO}$-filaments with those of the gas detected in absorption and emission in the direction of extragalactic continuum sources by Liszt \& Lucas (1998). Their lines of sight perform a random sampling of the diffuse molecular medium and some of them sample the same kind of gas as that emitting here in the ${ }^{12} \mathrm{CO}$ wings. The five absorption components with $N\left({ }^{12} \mathrm{CO}\right) \approx 10^{15} \mathrm{~cm}^{-2}$, observed in the directions of B0355, B0224, B1730, B2013 and B2251, are optically thin $\left(\tau_{12}<1\right)$, with velocity dispersions in the range 0.5 to $1 \mathrm{~km} \mathrm{~s}^{-1}$, and have a ${ }^{12} \mathrm{CO}$ line temperature of 0.5 to $1.8 \mathrm{~K}$ when observed in emission. These properties are all very similar to those of the optically thin ${ }^{12} \mathrm{CO}$-filaments (see Table 5). Moreover, the non detection, in the boxes of Fig. 7, of the ${ }^{13} \mathrm{CO}$ line above the rms noise level, implies that $N\left({ }^{12} \mathrm{CO}\right) / N\left({ }^{13} \mathrm{CO}\right)>30$ with $N\left({ }^{12} \mathrm{CO}\right)=0.9-$ $1.4 \times 10^{15} \mathrm{~cm}^{-2}$ (Sect. 5.3), a value that delicately fits among their data points at $N\left({ }^{12} \mathrm{CO}\right) \approx 10^{15} \mathrm{~cm}^{-2}$. Our data set therefore possibly reveals the remarkable filamentary morphology of the gas seen in absorption by Liszt \& Lucas (1998) and may shed light on the processes driving $\mathrm{CO}$ formation in translucent gas. There is no way, though, at that stage, to determine whether CO is being formed or photo-dissociated in these far-wings. We postpone to Papers III and IV the discussion of the possible connexion between these structures and turbulence dissipation. In this framework, such structures are sites of a warm specific chemistry, fed by bursts of turbulent dissipation, that has been modeled by Joulain et al. (1998) and Falgarone et al. (2006).

The present study allows us to compare the $\mathrm{CO}$ cooling rate of the optically thin ${ }^{12} \mathrm{CO}$-filaments with that of the whole field on average. We estimate the $\mathrm{CO}$ cooling rate with the first four ${ }^{12} \mathrm{CO}$ rotational transitions, $\Lambda_{\mathrm{CO}}=\sum_{u=1,4} 8 \pi k v_{u l}^{3} / c^{3} \times T_{u l} \Delta v / D$. For the ${ }^{12} \mathrm{CO}$-filaments, the LVG solutions of Sect. 5.3 give

$\Lambda_{\mathrm{CO}, \text { wing }}=2-4 \times 10^{-24} \frac{\Delta v}{0.35 \mathrm{~km} \mathrm{~s}^{-1}}\left(\frac{D}{0.03 \mathrm{pc}}\right)^{-1} \mathrm{erg} \mathrm{s}^{-1} \mathrm{~cm}^{-3}(5)$

increasing steadily from $2 \times 10^{-24}$ at $T_{\text {kin }}=25 \mathrm{~K}$ to $4 \times$ $10^{-24} \mathrm{erg} \mathrm{s}^{-1} \mathrm{~cm}^{-3}$ at $T_{\text {kin }}=400 \mathrm{~K}$. On average for the whole field, we use the (1-0) and (2-1) emissions from Table 2 and apply the line ratio $(3-2) /(2-1)=0.41$ and $(4-3) /(2-$ $1)=0.12$ found by Falgarone \& Phillips (1996) for the
${ }^{12} \mathrm{CO}$ line core in a similar environment, and obtain $\Lambda_{\mathrm{CO}}=$ $10^{-24}\left(W_{10} / 10.9 \mathrm{~K} \mathrm{~km} \mathrm{~s}^{-1}\right)(D / 1 \mathrm{pc})^{-1} \mathrm{erg} \mathrm{cm}^{-3} \mathrm{~s}^{-1}$ (see Table 2). Thus, under the assumption that the ratio of the depths along the line of sight are the same as the ratio of their projected dimensions, we find that the ${ }^{12} \mathrm{CO}$-filaments radiate 2 to 4 times more in the $\mathrm{CO}$ lines than the rest of the field while they contribute to about $10 \%$ of its mass.

\subsection{Importance of magnetic fields in the gas dynamics}

The analysis of the orientations of the structures shows that the ${ }^{12} \mathrm{CO}$ substructures and the ${ }^{13} \mathrm{CO}$-filaments have most probable position angles $\mathrm{PA}=100 \pm 40$ and $108 \pm 25^{\circ}$ respectively. Absorption measurements of the light from a background star, located $1.6^{\circ}(\approx 4 \mathrm{pc})$ in the North of the dense core, provide the orientation of the magnetic fields projected on the plane of the sky $\left(B_{\text {pos }}\right)$ : the polarization angle is $108 \pm 19^{\circ}$ (Heiles 2000). The similarity between the dominant orientation of the structures and the direction of $B_{\text {pos }}$ measured in the neighborhood suggests that the magnetic fields are responsible for the observed anisotropy.

Chandrasekhar \& Fermi (1953) proposed that the dispersion of the orientation of the magnetic field lines in a known turbulent flow could yield an estimate of the strength of the fields. This method has been tested and calibrated on numerical simulations of MHD turbulence by Ostriker et al. (2001) and Crutcher (2005), and numerically provides the plane-of-the-sky field strength as a function of the properties of the ambient turbulence and the observed fluctuations of the polarization angle:

$B_{\mathrm{pos}} \approx 6.6{\overline{n_{\mathrm{H}}}}^{1 / 2} \frac{\Delta v}{\delta \phi} \quad \mu \mathrm{G}$

where $\overline{n_{\mathrm{H}}}$ is the total $\mathrm{H}$ density in $\mathrm{cm}^{-3}, \Delta v$ is the $F W H M$ of emission lines in $\mathrm{km} \mathrm{s}^{-1}$, and $\delta \phi$ is the position angle dispersion in degrees. Ostriker et al. (2001) have shown that this method is reliable for $\delta \phi<25^{\circ}$. This can be understood as follows: from the previous relation, the ratio of the Alfvén velocity (measured with $\left.B_{\text {pos }}\right)$ to the most probable velocity in a turbulent flow $(\bar{v}=$ $\sqrt{2} \Delta v / 2.35)$ is

$v_{\mathrm{A}} / \bar{v}>v_{\mathrm{A}, \mathrm{pos}} / \bar{v}=20^{\circ} / \delta \phi$.

Fluctuation angles much larger than $20^{\circ}$ correspond to superAlfvénic flows where the magnetic field lines become so tangled that the concept of fluctuations no longer holds.

In this field, there is no direct measurement of the fluctuations of the polarization position angle of the magnetic fields over scales as small as $0.03 \mathrm{pc}$. Because partially ionized gas preferentially flows along the field lines, we assume that the fluctuations of the position angle of the ${ }^{12} \mathrm{CO}$-filaments reflect the entanglement of the magnetic field lines. From Sect. 4.2, the dispersion of the orientations of the ${ }^{12} \mathrm{CO}$-filaments is $\delta \phi=25^{\circ}$, making the Chandrasekhar \& Fermi (1953) method reliable. The turbulence in the envelope is therefore in the trans-Alfvénic regime characterized by a Alfvén Mach number $M_{A}=\bar{v} / v_{\mathrm{A}}<$ 1.25. From Eq. (6), assuming an average density $\overline{n_{\mathrm{H}}}=N_{\mathrm{H}} / D \approx$ $500 \mathrm{~cm}^{-3}, B_{\text {pos }}=6.6{\overline{n_{\mathrm{H}}}}^{1 / 2}\left(\Delta v / 2.5 \mathrm{~km} \mathrm{~s}^{-1}\right) / \delta \phi \approx 15 \mu \mathrm{G}$ or, if $\boldsymbol{B}$ is randomly distributed, $B=1.3 B_{\mathrm{pos}} \approx 20 \mu \mathrm{G}$.

The dynamical importance of the magnetic fields is further supported by the conspicuous oscillating velocity pattern disclosed in one ${ }^{13} \mathrm{CO}$-filament, interpreted as gas freely flowing along helical magnetic field lines (see Sect. 6.3). The orientation of the helix $(\mathrm{PA} \approx 0)$ differs from the general orientation of the 
tail sub-structures by $\approx 80^{\circ}$, only $2 \sigma$ of the probability distribution function of PA (Fig. 10). We cannot rule out that the material in the helix is unrelated to that in the dense core tail. But it is precisely because its orientation differs significantly from the average that we have been able to detect it and detail the dynamics of the gas there.

These results may be put in perspective with star formation. The $1 M_{\odot}$ dense core in the field harbors two condensations of 0.13 and $0.19 M_{\odot}$ which are gravitationally bound according to Heithausen (2002). Inward motions have been detected in one of them (Heithausen 1999) suggesting infalling motions possibly due to the onset of gravitational instability, and this dense core may thus be a pre-star-forming core. Magnetic fields are found to play a dominant role in the dynamics of its environment, imprinting its configuration to the gas structure and possibly channeling the gas in the tail onto the core. Our observations thus suggest that magnetic fields play a significant role during the building phase of this dense core.

\section{Summary}

As observed in the present study (i.e. over a large dynamic range of scales and at high angular resolution), the translucent environment of a low mass dense core is found to harbor a large diversity of gas densities and temperatures and a wealth of space-velocity structures. There is a predominance of filamentary structures (i.e. structures with aspect ratio as high as 20) which would have been overlooked in smaller or less sensitive maps. In particular, the $1 M_{\odot}$ dense core itself is not isolated but connected to a long tail of dense gas, at least twice as massive as the core, that may be seen as a possible mass reservoir for the core growth. This tail is highly turbulent and sub-structured into many thinner entities that are shown to be almost pure velocity structures. A more dilute envelope comprises a network of narrow $(0.03 \mathrm{pc}$ in diameter) filamentary structures, optically thin in the ${ }^{12} \mathrm{CO}(1-0)$ line, found to be warmer than $25 \mathrm{~K}$ and more dilute than $1000 \mathrm{~cm}^{-3}$. As a whole, the optically thin gas amounts to about $10 \%$ of the gas mass in the field.

It is unlikely that these optically thin ${ }^{12} \mathrm{CO}$-filaments would be strong shocks because they consist of dilute gas. They radiate in the CO lines a few times more than the rest of the field on average and are good candidates for the long-searched dissipative structures of turbulence.

We also find that the structures, either dense or dilute, are preferentially oriented in the direction of the magnetic field, measured a few parsecs away. Magnetic fields are therefore expected to play an important role in the dynamical evolution of the dense core environment, a point that we confirm by deriving the field intensity from the fluctuations of the orientation of the optically thin filaments. In the envelope, we find that the turbulent motions are in the trans-Alfvénic regime.

This work provides a global and detailed view of a dense core environment: it shows how and over which scales the small core is connected to its parsec-scale environment. It also reveals for the first time the morphology and the gas properties of structures that may be sites of turbulence dissipation. Whether these structures play a role in the formation and growth of the nearby dense core is a question that warrants further observations of a variety of core environments made with large dynamic range, as well as confrontations with numerical simulations of star formation in turbulent and magnetized environment.

Acknowledgements. We thank the referee for his careful reading of the manuscript and for his fruitful suggestions. We are grateful to Malcolm

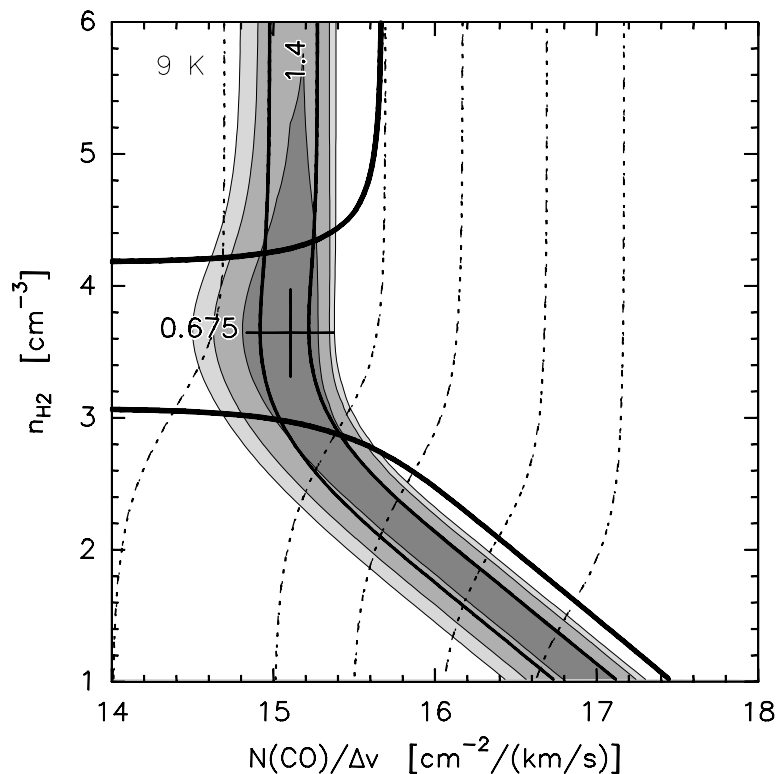

Fig. A.1. $\mathrm{LVG}$ calculations at $T_{\text {kin }}=9 \mathrm{~K}$ for the dense core tail gas. Constraints from Table 3 on ${ }^{13} \mathrm{CO}(1-0)\left(T_{\mathrm{mb}}=1.6 \pm 0.6 \mathrm{~K}\right)$ and ${ }^{13} \mathrm{CO}(2-1) /(1-0)(=0.35-1$, thick $)$ are indicated with full lines. The grey scale shows $\chi^{2}$ with contours at $1,1.7$ and $2 \sigma$ levels. Dot-dashed lines show the delineates the center line opacity of the ${ }^{13} \mathrm{CO}(1-0)$ : $\tau_{13}=0.1,1,3,10$ and 30 (from left to right). The cross locates the minimum- $\chi^{2}$ solution.

Walmsley for his suggestion to use automatic procedures to quantify the orientations of the structures.

\section{Appendix A: LVG calculations}

Figure A.2 shows the results of LVG calculations at two temperatures, 10 and $100 \mathrm{~K}$. The constraints are $T_{12}(1-0)=2.3 \pm 0.3 \mathrm{~K}$ and $R_{12}=0.7 \pm 0.1$. The Sets of solutions are derived by minimizing the $\chi^{2}$ defined by

$\chi^{2}=\sum_{k=1}^{N}\left(\frac{X_{k}-O_{k}}{\sigma_{k}}\right)^{2}$

where $N$ is the number of constraints (here $N=2$ ), $X_{k}$ and $O_{k}$ are the LVG and observed values of observable $k$, and $\sigma_{k}$ the dispersion around $O_{k}$. Confidence levels are then deduced from the variations $\Delta \chi^{2}$ above the minimum $\chi_{\min }^{2}$ of the $\chi^{2}$ hypersurface. With 2 degrees of freedom, a $1 \sigma$ confidence level $(68.3 \%)$ corresponds to $\Delta \chi^{2}=2.3$, and a $2 \sigma$ level $(95.5 \%)$ corresponds to $\Delta \chi^{2}=6.14$.

\section{Appendix B: Helical flow modelling}

In the helix frame $R=(O, O x, O y, O z)$, we consider gas cells positions given by

$\mathbf{X}(s)=\left[\begin{array}{c}v_{0} s \\ r_{1} \cos (\omega s) \\ r_{2} \sin (\omega s)\end{array}\right]$

where the helix is along the $x$-axis, $\lambda=2 \pi / \omega$ is its wavelength, and $s$ is the curvilinear coordinate along the helix. The observer frame is $R_{0}=\left(O x_{0}, O y_{0}, O z_{0}\right)$, and the plane of the sky is $\left(O y_{0}, O z_{0}\right)$. The $x$-axis is first rotated by $\theta$ with respect to $O y_{0}$, 

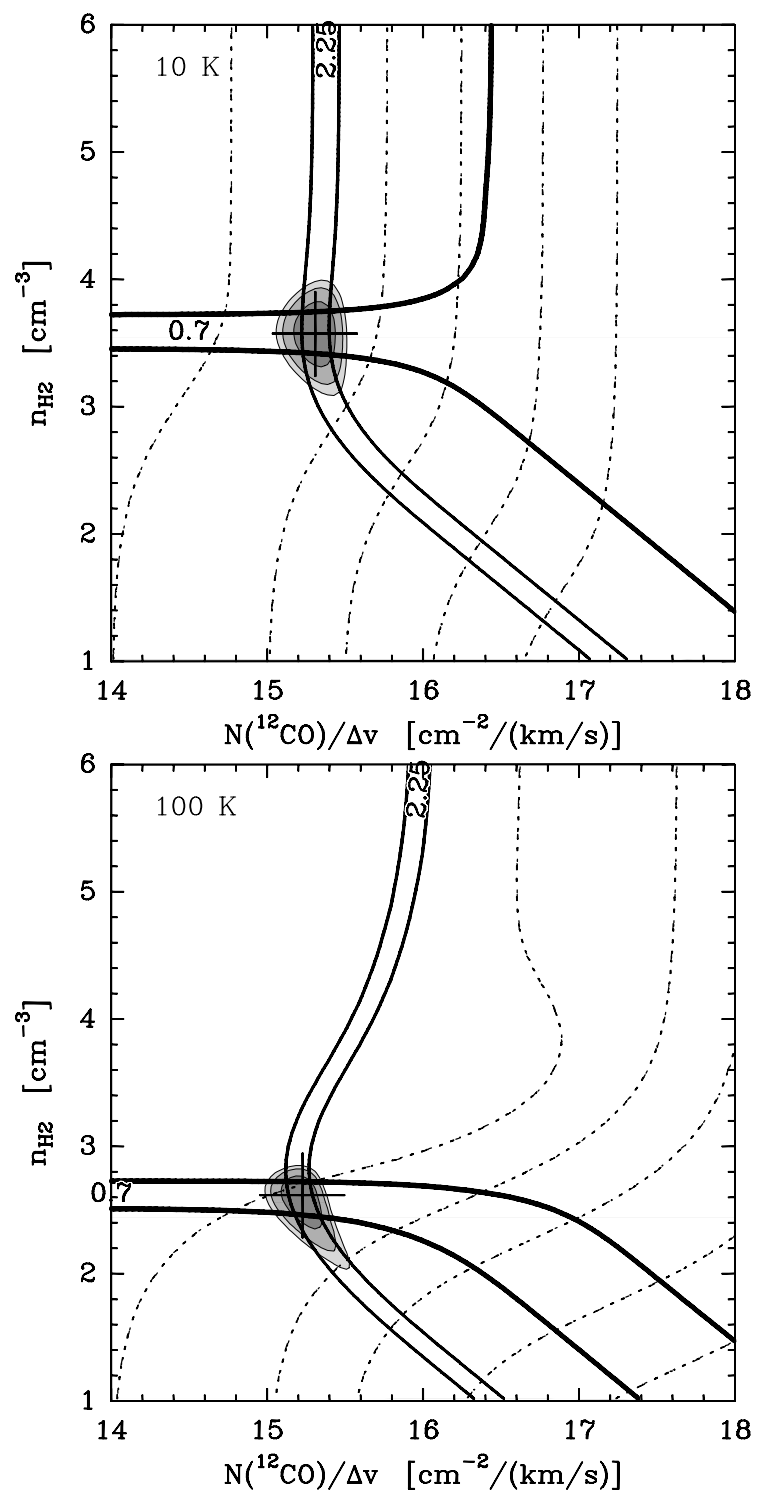

Fig. A.2. $\mathrm{LVG}$ calculations for the ${ }^{12} \mathrm{CO}$-wings (see Sect. 5.3) at two kinetic temperatures $(10$ and $100 \mathrm{~K})$. Full lines give the ${ }^{12} \mathrm{CO}(1-0)$ mainbeam temperatures and $R_{12}$ (thick). $\chi^{2}$-contours and dot-dashed (opacity) lines are as in Fig. A.1.

and then by $\phi$ with respect to $O z_{0}$. The rotation matrix is

$$
\begin{aligned}
\mathbf{R}(\theta, \phi) & =\mathbf{R}_{\phi, O z} \cdot \mathbf{R}_{\theta, O y} \\
& =\left[\begin{array}{ccc}
\cos \phi \cos \theta & -\sin \phi & -\cos \phi \sin \theta \\
\sin \phi \cos \theta & \cos \phi & -\sin \phi \sin \theta \\
\sin \theta & 0 & \cos \theta
\end{array}\right]
\end{aligned}
$$

which gives the helix coordinates in $R_{0}$ :

$\mathbf{X}_{0}(s)=\mathbf{R}(\theta, \phi) \mathbf{X}(s)$

or, by developing the matrix product,

$$
\mathbf{X}_{0}(s)=\left[\begin{array}{c}
x(s) \cos \phi \cos \theta-y(s) \sin \phi-z(s) \cos \phi \sin \theta \\
x(s) \sin \phi \cos \theta+y(s) \cos \phi-z(s) \sin \phi \sin \theta \\
x(s) \sin \theta+z(s) \cos \theta
\end{array}\right] .
$$

The projected velocity along the los is simply the $z_{0}$ component of the first derivative of $\mathbf{X}_{0}(s)$

$$
\begin{aligned}
v_{z, 0}(s) & =v_{x}(s) \sin \theta+v_{z}(s) \cos \theta \\
& =v_{0} \sin \theta+r_{2} \omega \cos (\omega t) \cos \theta .
\end{aligned}
$$

The helix is then sampled on a regular grid, and smoothed to the desired spatial resolution. A Gaussian emission spectrum is produced at each point in the structure, centered at the line-ofsight velocity $v_{z, 0}(s)$, whose linewidth is independent of the position along the helix. The final product is a position-positionvelocity datacube, which is compared to the observations.

\section{References}

Abergel, A., Boulanger, F., Mizuno, A., \& Fukui, Y. 1994, ApJ, 423, L59 Bensch, F., Stutzki, J., \& Heithausen, A. 2001, A\&A, 365, 285

Bernard, J. P., Abergel, A., Ristorcelli, I., et al. 1999, A\&A, 347, 640

Cambrésy, L., Boulanger, F., Lagache, G., \& Stepnik, B. 2001, A\&A, 375, 999 Chandrasekhar, S., \& Fermi, E. 1953, ApJ, 118, 113

Crutcher, R. M. 2005, in Magnetic Fields in the Universe: From Laboratory and Stars to Primordial Structures., ed. E. M. de Gouveia dal Pino, G. Lugones, \& A. Lazarian, AIP Conf. Proc. 784, 129

Emerson, D. T., \& Gräve, R. 1988, A\&A, 190, 353

Falgarone, E. \& Phillips, T. G. 1996, ApJ, 472, 191

Falgarone, E., Phillips, T. G., \& Walker, C. K. 1991, ApJ, 378, 186

Falgarone, E., Lis, D. C., Phillips, T. G., et al. 1994, ApJ, 436, 728

Falgarone, E., Panis, J.-F., Heithausen, A., et al. 1998, A\&A, 331, 669

Falgarone, E., Pety, J., \& Phillips, T. G. 2001, ApJ, 555, 178

Falgarone, E., Pineau Des Forêts, G., Hily-Blant, P., \& Schilke, P. 2006, A\&A, 452,511

Falgarone, E., Pety, J., \& Hily-Blant, P. 2007, in prep.

Fiege, J. D. \& Pudritz, R. E. 2000a, MNRAS, 311, 85

Flower, D. 2001, J. Phys. B: At. Mol. Opt., 534, 2731

Fuller, G. A. \& Myers, P. C. 1992, ApJ, 384, 523

Gerin, M., Falgarone, E., Joulain, K., et al. 1997, A\&A, 318, 579

Greve, A., Kramer, C., \& Wild, W. 1998, A\&AS, 133, 271

Grossmann, V., Meyerdierks, H., Mebold, U., \& Heithausen, A. 1990, A\&A, 240,400

Haikala, L. K., Harju, J., Mattila, K., \& Toriseva, M. 2005, A\&A, 431, 149

Heiles, C. 2000, AJ, 119, 923

Heithausen, A. 1999, A\&A, 349, L53

Heithausen, A. 2002, A\&A, 393, L41

Heithausen, A., Stacy, J. G., de Vries, H. W., Mebold, U., \& Thaddeus, P. 1993, A\&A, 268, 265

Heithausen, A. \& Thaddeus, P. 1990, ApJ, 353, L49

Hily-Blant, P. 2004, Ph.D. Thesis, Université d'Orsay (France)

Hily-Blant, P., Falgarone, E., \& Pety, J. 2007, submitted

Hily-Blant, P., Falgarone, E., Pineau Des Forêts, G., \& Phillips T., G. 2004, Ap\&SS, 292, 285

Hily-Blant, P., Teyssier, D., Philipp, S., \& Güsten, R. 2005, A\&A, 440, 909

Johnstone, D., Wilson, C. D., Moriarty-Schieven, G., et al. 2000, ApJ, 545, 327

Joulain, K., Falgarone, E., Des Forêts, G. P., \& Flower, D. 1998, A\&A, 340, 241

Langer, W. D., Graedel, T. E., Frerking, M. A., \& Armentrout, P. B. 1984, ApJ, 277, 581

Lis, D. C., Pety, J., Phillips, T. G., \& Falgarone, E. 1996, ApJ, 463, 623

Liszt, H. S. \& Lucas, R. 1998, A\&A, 339, 561

Loren, R. B. 1989a, ApJ, 338, 902

Martin, H. M., Hills, R. E., \& Sanders, D. B. 1984, MNRAS, 208, 35

McKee, C. F., Storey, J. W. V., Watson, D. M., \& Green, S. 1982, ApJ, 259, 647

Mizuno, A., Onishi, T., Yonekura, Y., et al. 1995, ApJ, 445, L161

Onishi, T., Kawamura, A., Abe, R., et al. 1999, PASJ, 51, 871

Ostriker, E. C., Stone, J. M., \& Gammie, C. F. 2001, ApJ, 546, 980

Padoan, P., Cambrésy, L., \& Langer, W. 2002, ApJ, 580, L57

Padoan, P., Juvela, M., Bally, J., \& Nordlund, A. 1998, ApJ, 504, 300

Perryman, M. A. C. \& ESA. 1997, The HIPPARCOS and TYCHO cata-

logues. Astrometric and photometric star catalogues derived from the ESA HIPPARCOS Space Astrometry Mission (Noordwijk, Netherlands: ESA)

Pety, J. \& Falgarone, E. 2000, A\&A, 356, 279

Pety, J. \& Falgarone, E. 2003, A\&A, 412, 417

Sofia, U. J., Lauroesch, J. T., Meyer, D. M., \& Cartledge, S. I. B. 2004, ApJ, 605, 272

Stutzki, J. \& Güsten, R. 1990, ApJ, 356, 513

Tauber, J. A., Goldsmith, P. F., \& Dickman, R. L. 1991, ApJ, 375, 635

Teixeira, P. S., Lada, C. J., \& Alves, J. F. 2005, ApJ, 629, 276

Williams, J. P., de Geus, E. J., \& Blitz, L. 1994, ApJ, 428, 693

Zagury, F., Boulanger, F., \& Banchet, V. 1999, A\&A, 352, 645 